\title{
Isomorphic Universality and the Number of Pairwise Nonisomorphic Models in the Class of Banach Spaces
}

\author{
Mirna Džamonja \\ School of Mathematics, University of East Anglia, Norwich NR4 7TJ, UK \\ Correspondence should be addressed to Mirna Džamonja; h020@uea.ac.uk
}

Received 7 December 2013; Revised 22 March 2014; Accepted 30 March 2014; Published 20 May 2014

Academic Editor: S. A. Mohiuddine

Copyright (C) 2014 Mirna Džamonja. This is an open access article distributed under the Creative Commons Attribution License, which permits unrestricted use, distribution, and reproduction in any medium, provided the original work is properly cited.

\begin{abstract}
We develop the framework of natural spaces to study isomorphic embeddings of Banach spaces. We then use it to show that a sufficient failure of the generalized continuum hypothesis implies that the universality number of Banach spaces of a given density under a certain kind of positive embedding (very positive embedding) is high. An example of a very positive embedding is a positive onto embedding between $C(K)$ and $C(L)$ for 0 -dimensional $K$ and $L$ such that the following requirement holds for all $h \neq 0$ and $f \geq 0$ in $C(K)$ : if $0 \leq T h \leq T f$, then there are constants $a \neq 0$ and $b$ with $0 \leq a \cdot h+b \leq f$ and $a \cdot h+b \neq 0$.
\end{abstract}

\section{Introduction}

In this paper we join a recent trend that has seen a combination of model theory and set theory address questions coming from analysis and topology. Examples are the spectacular proof by Malliaris and Shelah of the $\mathfrak{p}=t$ in [1] or more directly connected to this paper, work by Shelah and Usvyatsov on the isometric universality of Banach spaces [2], which will be mentioned in more detail below. We are specifically interested in the isomorphic embeddings of Banach spaces, in particular in the universality number of this class. This is the minimal number of the Banach spaces of a given density which isomorphically embed all the other spaces of the same density, allowing, depending on the context, the embeddings or the spaces to have extra properties. In many contexts, for example, when working just with plain isomorphic embeddings, it suffices to work with spaces of the form $C(K)$ and even to assume that $K$ is 0 -dimensional and so of the form $\operatorname{St}(\mathfrak{A})$ where $\mathfrak{A}$ is a Boolean algebra and $\operatorname{St}(\mathfrak{A})$ its Stone space. This is because every Banach space of a given density embeds isometrically into one of the form $C(\operatorname{St}(\mathfrak{A}))$ with the same density. In the main body of this paper we concentrate on the spaces of the form $C(\operatorname{St}(\mathfrak{A}))$.

The topic of universality of Banach spaces has already received a significant input from set theory, notably in the work of Brech and Koszmider [3, 4], which will be reviewed below. The new element we bring is the study of the topic not only by set-theoretic methods such as forcing, but also by the methods coming from classification theory in model theory and pcf theory. These ideas were explored in the context of isometric embeddings in [2], but the methods applicable to isometries do not at all apply in the context of isomorphic embeddings; hence we have needed to construct a new framework. Using this framework we are able to provide a template (Theorem 16(1)) of results which state that, for an uncountable cardinal number $\lambda$ under certain cardinal arithmetic assumptions, there is no universal Banach space of density $\lambda$, under certain kinds of isomorphic embeddings. The general kind of embeddings considered in the template is called very positive embeddings and it includes many natural examples of embeddings. One is presented in the following theorem which easily follows from Theorem 16(1) (see Section 5.3).

Theorem 1. Suppose that $\theta$ and $\lambda$ are two regular cardinals with

$$
\aleph_{2} \leq \theta<\theta^{+}<\lambda<2^{\theta},
$$

and that $(\forall \kappa<\lambda) \kappa^{\aleph_{0}}<\lambda$.

Then the minimal number of spaces of the form $C(S t(\mathfrak{H}))$ of density $\lambda$ needed to embed all Banach spaces of the form $C(\operatorname{St}(\mathfrak{B}))$ of density $\lambda$ by an embeddings $T$ satisfying the following conditions (i)-(iii) is $2^{\theta}$, where 
(i) $T$ is positive; that is, $f \leq g \Rightarrow T f \leq T g$;

(ii) $T$ is onto;

(iii) if $0 \leq T h \leq T f, h \neq 0$, and $f \geq 0$, then there are constants $a \neq 0, b$ such that $0 \leq a \cdot h+b \leq f$ and $a \cdot h+b \neq 0$.

In particular there is no surjectively universal space $C(S t(\mathfrak{A}))$ of density $\lambda$, under the embeddings satisfying (i)-(iii).

It would of course be desirable to weaken the condition of very positivity. In recent work, Shelah [5] introduces a modeltheoretic property called the olive property which generalizes the model-theoretic tools that can be used to apply the method of invariants which were used in the context of linear orders in [6] and which are necessary for [2] and for this work. The restriction to very positivity in our work basically comes from the connection of the invariants to linear orders. Therefore it is a promising direction for future work to find a Banach space isomorphism context where one could use the olive property in place of the order.

An additional consideration of the paper is the subject of the number of pairwise nonisomorphic Banach spaces of a given density. For example, the celebrated Kaplansky theorem [7] shows that if $C(K)$ and $C(L)$ admit a bijective isomorphism which also preserves pointwise order, then $K$ and $L$ are homeomorphic. Coupled with model-theoretic results which show that for every uncountable $\kappa$ there are $2^{\kappa}$ pairwise nonisomorphic Boolean algebras of size $\kappa$ and with the Stone representation theorem, this gives that there are $2^{\kappa}$ pairwise non-order-isomorphic Banach spaces of density $\kappa$. It follows from our work, as we show in Theorem 16(2), that the same is true under the weaker assumption of very positive embeddings (that the assumption of very positivity is strictly weaker than the assumption of preserving order follows from our examples in Section 5). A very general modeltheoretic study of the properties which lead to a large number of pairwise nonisomorphic models in metric structures is undertaken by Shelah and Usvyatsov in [8] and in the future it may yield more general results about nonisomorphic Banach spaces.

We should finish this introduction by mentioning that for uncountable $\lambda$ the successor of a regular it is not difficult to construct specific models of set theory in which there are no isomorphically universal Banach spaces of density $\lambda$; for example, the classical Cohen model will do (paper [9] addresses this and finer versions of it). The point of Theorem 16 is that it is not a result which is true just in some specifically constructed model; it is a result which holds as soon as certain cardinal arithmetic assumptions are fulfilled. Another remark is that on the basis of what is known in the literature and what we obtain here, no known result differentiates between the universality number of Banach spaces of a given density under isometries or under isomorphisms. Furthermore, it is not known how to differentiate them from the universality number of Boolean algebras.

\section{Background}

For a quasi-ordered class $(\mathscr{M}, \leq)$, the universality number is defined as the smallest size of $\mathscr{N} \subseteq \mathscr{M}$ such that for every $M \in$ $\mathscr{M}$ there is $N \in \mathscr{N}$ such that $M \leq N$. In Banach space theory we find many examples of classes whose universality numbers have been studied, with respect to isomorphic, isometric, and other kinds of embeddings. A classical result by Banach [10, page 185$]$ shows that $C([0,1])$ is isometrically universal for all separable Banach spaces.

For the nonseparable case, the situation is more complex. The cardinal arithmetic assumption GCH automatically gives one universal model for each uncountable density, as explained below. Specific models of the failure of GCH were studied by Brech and Koszmider [3] who considered Banach spaces of density the continuum and proved that in the Cohen model for $\aleph_{2}$ many Cohen reals there is an isomorphically universal Banach space of density $\mathfrak{c}=\aleph_{2}$. In [9] there are negative universality results in Cohen and Cohen-like models; for example, the isomorphic universality number for Banach spaces of density $\aleph_{1}$ is $\aleph_{2}$ in the Cohen model and moreover one Cohen real adds a w.c.g. Banach space of density $\aleph_{1}$ which does not embed into any Banach space with a dense set of size $\aleph_{1}$ in the ground model. In [4], which studies w.c.g. Banach spaces, it is stated (page 1268), without proof, that Koszmider and Thompson noted that a version of the proof from [3] gives a model where there is no isomorphically universal Banach space of density $\aleph_{1}$. Let us briefly explain the known positive universality results in the context of GCH. Throughout, $\kappa$ stands for an infinite cardinal.

By combining the Stone duality theorem, the fact that any Banach space $X$ is isometric to a subspace of $C\left(B_{X^{*}}\right)$ and that $B_{X^{*}}$ has a totally disconnected continuous preimage, Brech and Koszmider proved the following.

Fact 1 (see [3], Fact 1.1). (1) The universality number of the class of Boolean algebras of size $\kappa$ is greater or equal to the universality number of the class of Banach spaces of density $\kappa$ with isometric embeddings, which is greater or equal than the universality number of the class of Banach spaces of density $\kappa$ with isomorphic embeddings.

(2) The class of spaces of the form $C(\operatorname{St}(\mathfrak{A}))$ for $\mathfrak{A}$ a Boolean algebra of size $\kappa$ is isometrically universal for the class of Banach spaces of density $\kappa$, and in particular its universality number with either isometric or isomorphic embeddings is the same as the universality number of the whole class of Banach spaces of density $\kappa$.

Fact 1 is only interesting in the context of uncountable $\kappa$, since for $\kappa=\aleph_{0}$ we have a universal Boolean algebra as well as an isometrically universal Banach space, as explained above. On the other hand, it is known from the classical model theory (see [11] for saturated and special models) that in the presence of GCH there is a universal Boolean algebra at every uncountable cardinal, so the questions of universality for the above classes are interesting in the context of the failure of the relevant instances of GCH. Negative universality results for Boolean algebras are known to hold when $\mathrm{GCH}$ fails sufficiently by the work of Kojman and Shelah [6] and 
in Cohen-like extensions by the work of Shelah (see [6] for a proof). Shelah and Usvyatsov proved in [2] that, in the models where the negative universality results that were obtained for Boolean algebras in [6] hold, the same negative universality results hold for Banach spaces under isometric embeddings. The smallest cardinal at which these results can apply is $\aleph_{2}$. For example, if $\lambda$ is a regular cardinal greater than $\aleph_{1}$ but smaller than $2^{\aleph_{0}}$ (so $2^{\aleph_{0}} \geq \aleph_{3}$ ), there is no universal under isometries Banach space of density $\lambda$.

Conjecture 2. The universality number of the class of Banach spaces of density $\kappa$ with isomorphic embeddings is the same as the universality number of the class of Boolean algebras of size $\kappa$.

It follows from the above discussion that Conjecture 2 would improve Fact 1(1) and it would imply the negative universality results of Shelah and Usvyatsov. For all we know at this point Conjecture 2 could be a theorem of ZFC; that is, it is not known to fail at any $\kappa$ even consistently. A particular case of Conjecture 2 is the following Conjecture 3 , which summarizes the most interesting case from the point of view of Banach space theory.

Conjecture 3. The universality number of the class of Banach spaces of density $\kappa$ with isomorphic embeddings is the same as the universality number of the class of Banach spaces of density $\kappa$ with isometric embeddings.

There is a considerable amount of study of other kinds of embeddings of Banach spaces, but isometries and isomorphisms and our work will fit into that area. Sticking to the spaces of the form $C(K)$, among the classically studied isomorphic embeddings are those that preserve multiplication or the ones that preserve the pointwise order of functions. It is known for either one of them (Gelfand and Kolmogorov [12] for the former and Kaplansky [7] for the latter) that if they are onto, they actually characterize the topological structure of the space; that is, if $T: C(K) \rightarrow C(L)$ is an onto embeddings which either preserves multiplication or the pointwise order, then $K$ and $L$ are homeomorphic. We will show that, in moving from the order preserving onto assumption just a small bit, we no longer have the preservation of the homeomorphic structure, but under the assumption that GCH fails sufficiently, we do have a large number of pairwise nonisomorphic spaces and a large universality number.

We now finish the introduction by giving some background information for the readers less familiar with Banach space theory.

Definition 4. A Banach space is a normed vector space complete in the metric induced by the norm. A linear embeddings $T: X \rightarrow Y$ between Banach spaces is an isometry if for every $x \in X$, we have $\|x\|=\|T x\|$, where we use $T x$ to denote $T(x)$. A linear embeddings $T: X \rightarrow Y$ between Banach spaces is an isomorphism if there is a constant $D>0$ such that, for every $x \in X$, we have $(1 / D)\|x\| \leq\|T x\| \leq D\|x\|$.
Remark 5. Every isometry is an isomorphism. An isomorphism is in particular an injective continuous function, and in fact, a linear map $T$ is an isomorphism if and only if both $T$ and $T^{-1}$ are linear and continuous.

For $T$ an isomorphism, we define $\|T\| \stackrel{\text { def }}{=} \sup \{\|T(f)\|:$ $\|f\|=1\}$.

Throughout the paper letters $\mathfrak{A}$ and $\mathfrak{B}$ will be used for Boolean algebras, $\kappa, \lambda$ for infinite cardinals, and $K$ and $L$ for compact spaces. The space $C(K)$ is the space of all continuous real-valued functions on $K$ with the topology given by the supremum norm $\|f\| \stackrel{\text { def }}{=} \sup \{f(x): x \in K\}$. We will write $\mathrm{St}(\mathfrak{A})$ for the Stone space of a Boolean algebra $\mathfrak{A}$, which is defined as the space of all ultrafilters $\boldsymbol{u}$ on $\mathfrak{A}$ with the topology generated by sets $[a] \stackrel{\text { def }}{=}\{u: a \in u\}$ as a clopen basis. Let us note that Fact 1 implies.

Observation 1. The universality number of Banach spaces of density $\kappa$, under any kind of embeddings, is either 1 or $\geq \kappa^{+}$.

This is so because if for any $\alpha^{*} \in\left[1, \kappa^{+}\right)$, we had that $\left\{X_{\alpha}\right.$ : $\left.\alpha<\alpha^{*}\right\}$ were a universal family of Banach spaces of density $\kappa$, then we could assume that each $X_{\alpha}=C\left(\operatorname{St}\left(\mathfrak{A}_{\alpha}\right)\right)$ for some Boolean algebras $\mathfrak{A}_{\alpha}$ of size $\kappa$. Therefore we could find a single algebra $\mathfrak{A}$ of size $\kappa$ such that all $\mathfrak{A}_{\alpha}$ embed into it (simply by freely generating an algebra by a disjoint union of all $\mathfrak{A}_{\alpha}$ ) and hence $C(\operatorname{St}(\mathfrak{U}))$ would be a single universal Banach space of density $\kappa$.

\section{Natural Spaces of Functions}

Our methods will involve a combination of model theory, set theory, and Banach space theory. In this section we introduce a simple model-theoretic structure which will be used to achieve that mixture of methods.

Suppose that $\mathfrak{A}$ is a Boolean algebra. We will associate to it a structure whose role is to represent the space $C(K)$, where $K$ is the Stone space of $\mathfrak{A}, K=\operatorname{St}(\mathfrak{A})$. The idea is as follows. We are interested in the set of all simple functions with rational coefficients defined on $K$, so functions of the type $\sum_{i \leq n} q_{i} \chi_{\left[a_{i}\right]}$, where each $q_{i}$ is rational, $a_{i} \in \mathfrak{A}$, and $\left[a_{i}\right]$ denotes the basic clopen set in $K$ determined by $a_{i}$. Every element of $C(K)$ is the limit of a sequence of such functions, since the limits of such sequences form exactly the class of Lebesgue integrable functions, which of course includes $C(K)$. Let us then consider the vector space freely generated by $\mathfrak{A}$ over $\mathbb{Q}$, call it $V=V(\mathfrak{A})$ (this vector space figures in [3] with the notation $C_{\mathbb{Q}}(\mathfrak{A})$ and is considered in a different context). Hence every simple function on $K$ with rational coefficients corresponds uniquely to an element of $V$, via an identification of each $a \in \mathfrak{A}$ with $\chi_{[a]}$. Using coordinatwise addition and scalar multiplication, the product $W=V^{\omega}$ becomes a vector space. Any function $f$ in $C(K)$ can be identified with an element of this vector space, namely, a sequence of simple rational functions whose limit is $f$, and hence $C(K)$ can be identified with a subset of $W$.

To encapsulate this discussion we will work with vector spaces with rational coefficients and with two distinguished 
unary predicates $C, C_{0}$ satisfying $C_{0} \subseteq C$. With our motivation in mind, we will call them function spaces. If such a space $\left(V, C, C_{0}\right)$ is the space of sequences of simple rational functions over a Stone space $K=\mathrm{St}(\mathfrak{U})$ and $C, C_{0}$ correspond, respectively, to the set of such sequences which converge or converge to 0 , then we call $\left(V, C, C_{0}\right)$ a natural space and we denote it by $N(\mathfrak{H})$. In spaces of the form $N(\mathfrak{H})$ for an element $\bar{f}$ of $C^{N(\mathfrak{A})}$ we define $\|\bar{f}\|$ as the norm in $C(\operatorname{St}(\mathfrak{A}))$ of the limit $f$ of $\bar{f}$. If $\phi$ is an embeddings between $N(\mathfrak{A})$ and $N(\mathfrak{B})$, we will say that $D>0$ is a constant of the embeddings if for every $\bar{f}$ of $C^{N(\mathfrak{2})}$, we have that $(1 / D) \cdot\|\bar{f}\| \leq\|\phi(\bar{f})\| \leq D \cdot\|\bar{f}\|$. Not every embeddings has such a constant, but we will only work with the ones which do.

We will mostly be interested in a specific case of the representation of continuous functions as limits of simple functions, given by the following observation.

Lemma 6. Suppose that $K=S t(\mathfrak{A})$ is the Stone space of a Boolean algebra $\mathfrak{A}$ and let $f \geq 0$ be a function in $C(K)$ with $\|f\| \leq D^{*}$ for some $D^{*}>0$. Then there is a sequence $\left\langle f_{n}: n<\right.$ $\omega\rangle$ of simple functions, where each $f_{n}$ is of the form $\Sigma_{i \leq n} q_{i} \chi_{\left[a_{i}\right]}$, with each $q_{i}$ rational in $\left(0, D^{*}\right]$ and $a_{i} \in \mathfrak{A}$, such that $f=$ $\lim f_{n}$.

Moreover, we can assume that for every $n, f_{n}$ is the sum of at most $n+1$ functions of the form $s \cdot \chi_{[a]}$.

Proof. By multiplying by a constant if necessary, we can assume that $D^{*}=1$. Functions of the form $\Sigma_{i \leq n} r_{i} \chi_{\left[a_{i}\right]}$ with each $r_{i}$ real, contain the constant function 1 , form an algebra, and separate the points of $K$; hence by the Stone-Weierstrass theorem, they form a dense subset of $C(K)$. Notice that every function $\Sigma_{i \leq n} r_{i} \chi_{\left[a_{i}\right]}$ can be, by changing the coefficients and the sets $a_{i}$ if necessary, represented in the form where all $\left[a_{i}\right]$ s are pairwise disjoint, so we can without loss of generality work only with such functions. Given $\varepsilon>0$ and $\Sigma_{i \leq n} r_{i} \chi_{\left[a_{i}\right]}$ with $a_{i}$ s disjoint, we can find for $i \leq n$ rational numbers $q_{i}$ with $\left|q_{i}-r_{i}\right|<\varepsilon$; hence the function $\Sigma_{i \leq n} r_{i} \chi_{\left[a_{i}\right]}$ is approximated within $\varepsilon$ by $\Sigma_{i \leq n} q_{i} \chi_{\left[a_{i}\right]}$, showing that also functions with rational coefficients and disjoint $a_{i}$ s are dense. Now given $f \geq 0$ a function in $C(K)$ with $\|f\| \leq 1$ and $\varepsilon>0$, let $\Sigma_{i \leq n} q_{i} \chi_{\left[a_{i}\right]}$ be a function with rational coefficients and disjoint $a_{i}$ s satisfying $\left\|f-\Sigma_{i \leq n} q_{i} \chi_{\left[a_{i}\right]}\right\|<\varepsilon$, recalling that the \|\| in $C(K)$ is the supremum norm. Define now

$$
s_{i}= \begin{cases}q_{i}, & \text { if } q_{i} \in[0,1], \\ 0, & \text { if } q_{i}<0, \\ 1, & \text { if } q_{i}>1,\end{cases}
$$

and consider the function $\Sigma_{i \leq n} s_{i} \chi_{\left[a_{i}\right]}$. We claim that $\| f-$ $\Sigma_{i \leq n} s_{i} \chi_{\left[a_{i}\right]} \|<\varepsilon$. By the assumption that $a_{i}$ s are disjoint, for any $x$, there is at most one $i=i(x)$ such that $x \in\left[a_{i}\right]$. If $x \notin \bigcup_{i \leq n}\left[a_{i}\right]$ or $s_{i(x)}=q_{i(x)}$, then $\left|f(x)-\Sigma_{i \leq n} s_{i} \chi_{\left[a_{i}\right]}(x)\right|=$ $\left|f(x)-\Sigma_{i \leq n} q_{i} \chi_{\left[a_{i}\right]}(x)\right|<\varepsilon$. If $x \in\left[a_{i}\right]$ and $q_{i}<0$, then $\left|f(x)-s_{i}\right|=f(x)<f(x)-q_{i}<\varepsilon$ as $f(x) \geq 0$. If $x \in\left[a_{i}\right]$ and $q_{i}>1$, then $\left|f(x)-s_{i}\right|=1-f(x)<q_{i}-f(x)<\varepsilon$ as $f(x) \leq 1$.

To finish the proof, we observe that given a sequence of functions $\left\langle f_{n}: n<\omega\right\rangle$ of the above form which converges to a function $f$, we can define by induction on $n, g_{0}=0, k(n)=$ $\min \left\{k: f_{k} \notin\left\{g_{0}, \ldots, g_{n-1}\right\}\right\}$ and $g_{n}=f_{k(n)}$ if the number of coefficients in $f_{k(n)}$ is $\leq n+1$, and $g_{n}=g_{n-1}$ otherwise. Hence $\left\langle g_{n}: 1 \leq n<\omega\right\rangle$ is the sequence $\left\langle f_{n}: 1 \leq n<\omega\right\rangle$ with possible repetitions of each element finitely many times and so $\lim _{n \rightarrow \infty} g_{n}=f$.

Definition 7. Suppose that $\left.\bar{f}=\left\langle f_{n}: n\right\rangle \omega\right\rangle$ is a sequence in $N(\mathfrak{A})$ and suppose that $\left.\bar{f}^{\prime}=\left\langle f_{n}^{\prime}: n\right\rangle \omega\right\rangle$ was obtained by first replacing each $f_{n}$ with an equivalent function $\Sigma_{i \leq n} q_{i} \chi_{\left[a_{i}\right]}$ with disjoint $a_{i}$ s and then replacing the coefficients $q_{i}$ by $s_{i}$ using the procedure described in the proof of Lemma 6 . We say that $\bar{f}^{\prime}$ is a top-up of $\bar{f}$.

Corollary 8. Suppose that $\mathfrak{A}$ and $\mathfrak{B}$ are Boolean algebras, and let $\mathscr{A}$ denote the linear subspace of $C^{N(\mathfrak{A})}$ spanned by the functions whose rational coefficients are in $[0,1]$. Then for every $\bar{f}=\left\langle f_{n}: n<\omega\right\rangle$ in $C^{N(\mathfrak{R})}$, there is $\bar{g} \in \mathscr{A}$ with $\lim _{n} f_{n}=\lim _{n} g_{n}$.

Proof. Let $f=\lim _{n} f_{n}$; hence $f$ can be written as $f=$ $f^{+}-f^{-}$where $f^{+}=\max \{f, 0\}$ and $f^{-}=\min \{f, 0\}$ are both continuous and positive. Therefore, by the closure of $\mathscr{A}$ under linear combinations, it suffices to prove the corollary in the case of $f \geq 0$. Let $D^{*}=\|f\|$, and we now apply Lemma 6 .

The point of these definitions is the connection between the embeddability in the class of spaces of the form $C(\operatorname{St}(\mathfrak{A}))$ and the class of function spaces. Namely, we have the following.

Theorem 9. Suppose that $\mathfrak{A}$ and $\mathfrak{B}$ are Boolean algebras, and let $\mathscr{A}$ denote the linear subspace of $C^{N(\mathfrak{O})}$ spanned by the family $\mathscr{A}^{\prime}$ of sequences $\left\langle f_{n}: n<\omega\right\rangle$ of simple functions whose coefficients are rationals in $[0,1]$ and which satisfy that each $f_{n}$ has at most $n+1$ many elements. Then if there is an isomorphic embeddings $T$ from $C(S t(\mathfrak{A}))$ to $C(S t(\mathfrak{B}))$, then there is an isomorphic embeddings $\phi$ from $\mathscr{A}$ to $N(\mathfrak{B})$ satisfying that for every $\bar{f}=\left\langle f_{n}: n<\omega\right\rangle$ in $\mathscr{A}$, if $f=\lim _{n \in \omega} f_{n}$, then $\lim _{n \in \omega} \phi(\bar{f})_{n}=T(f)$.

Proof. Let $T: C(\operatorname{St}(\mathfrak{A})) \rightarrow C(\operatorname{St}(\mathfrak{B}))$ be an isomorphic embeddings, so $\|T\|<\infty$. We intend to define an isomorphic embeddings $\phi$ from $\mathscr{A}$ to $N(\mathfrak{B})$. By linearity it is sufficient to work with the basis $\mathscr{A}^{\prime}$ of $\mathscr{A}$. Let us use the notation $\pi_{n}$ for the projection on the $n$th coordinate. First we define the action of $\phi$ on those $f \in \mathscr{A}^{\prime}$ which have the property that there is at most one $n$ such that $\pi_{n}(f)$ is not the identity zero function, and then $\pi_{n}(f)$ is a function of the form $\chi_{[a]}$ for some $a \in \mathfrak{A}$. If there is no such $n$ with $a \neq 0$, then we let $\phi(f)$ be the element of $N(\mathfrak{B})$ whose all projections $\pi_{n}$ are zero. Otherwise, let $n$ be such that $\pi_{n}(f) \neq 0$ and consider $T\left(\pi_{n}(f)\right)$, which is well defined. We have no reason to believe that $T\left(\pi_{n}(f)\right)$ is a simple function with rational coefficients. However, there is a function $F\left(\left(\pi_{n}(f)\right)\right)$ which is a simple function with rational coefficients and whose distance to $T\left(\pi_{n}(f)\right)$ in $C(\operatorname{St}(\mathfrak{B}))$ is less than $1 / 2^{n+1}$. We define $\phi(f)$ to be the unique element $g$ of $N(\mathfrak{B})$ such that the only $\pi_{m}(g)$ which is not identically zero is $\pi_{n}(g)$ and $\pi_{n}(g)=F\left(\left(\pi_{n}(f)\right)\right)$. 
Now suppose that $\bar{f} \in \mathscr{A}^{\prime}$ is such that for exactly one $n$, $\pi_{n}(\bar{f})$ is not identity zero, and $\pi_{n}(\bar{f})=\sum_{i=0}^{m} q_{i} \chi_{\left[a_{i}\right]}$ for some $a_{0}, \ldots, a_{m} \in \mathfrak{A}$ and some rational $q_{0}, \ldots, q_{m}$ in $[0,1]$. For $i \leq m$, let $\bar{f}_{i}$ be the element of $N(\mathfrak{A})$ whose $n$th projection is $\chi_{\left[a_{i}\right]}$ and all other projections are identity zero. Hence we have already defined $\phi\left(\bar{f}_{i}\right)$, and we let $\phi(\bar{f})=\sum_{i \leq m} q_{i} \phi\left(\bar{f}_{i}\right)$.

Finally suppose that $f=\left\langle f_{n}: n<\omega\right\rangle$ is any element of $\mathscr{A}^{\prime}$. Therefore for every $n$, we have already defined $g_{n}=$ $\phi\left(\left\langle 0, \ldots, f_{n}, 0, \ldots\right\rangle\right)$, where $f_{n}$ is on the $n$th coordinate. Let $\phi(f)=\left\langle g_{n}: n<\omega\right\rangle$. Hence we have defined a linear embeddings of $\mathscr{A}^{\prime}$ to $N(\mathfrak{B})$. We extend this embeddings to $\mathscr{A}$ by linearity. We need to check that this embeddings preserves $C$ and $C_{0}$, and again it suffices to concentrate on the basis $\mathscr{A}^{\prime}$. So suppose that $\bar{f}=\left\langle f_{n}: n<\omega\right\rangle$ is in $C^{N(\mathfrak{R})} \cap \mathscr{A}^{\prime}$, and let $\bar{g}=\left\langle g_{n}: n<\omega\right\rangle$ be its image under $\phi$. We will show that $\bar{g} \in C^{N(\mathfrak{B})}$ by showing that it is a Cauchy sequence. Let $n, m<\omega$; we will consider $\left\|g_{n}-g_{m}\right\|$. Let $f_{n}=\sum_{i \leq k} q_{i} \cdot \chi_{\left[a_{i}\right]}$ and $f_{m}=\sum_{j \leq l} r_{j} \cdot \chi_{\left[b_{j}\right]}$. We have

$$
\begin{aligned}
\left\|g_{n}-g_{m}\right\|= & \left\|\phi\left(f_{n}\right)-\phi\left(f_{m}\right)\right\| \\
\leq & \left\|\phi\left(f_{n}\right)-T\left(f_{n}\right)\right\|+\left\|T\left(f_{n}\right)-T\left(f_{m}\right)\right\| \\
& +\left\|T\left(f_{m}\right)-\phi\left(f_{m}\right)\right\| \\
\leq & \sum_{i \leq k}\left|q_{i}\right|\left\|\phi\left(\chi_{\left[a_{i}\right]}\right)-T\left(\chi_{\left[a_{i}\right]}\right)\right\|+\left\|T\left(f_{n}-f_{m}\right)\right\| \\
& +\sum_{j \leq l}\left|r_{j}\right|\left\|\phi\left(\chi_{\left[b_{j}\right]}\right)-T\left(\chi_{\left[b_{j}\right]}\right]\right\| \\
\leq & \frac{(k+1)}{2^{n+1}}+\|T\| \cdot\left\|f_{n}-f_{m}\right\|+\frac{(l+1)}{2^{m+1}},
\end{aligned}
$$

which goes to 0 as $n, m \rightarrow \infty$.

At the end suppose that $\bar{f}=\left\langle f_{n}: n\langle\omega\rangle\right.$ is in $C_{0}^{N(\mathfrak{R})} \cap \mathscr{A}^{\prime}$, and let $\bar{g}=\left\langle g_{n}: n<\omega\right\rangle$ be its image under $\phi$. By the definition of $\phi$, we have that $\left\|g_{n}\right\| \leq\left\|T\left(f_{n}\right)\right\|+(n+1) / 2^{n+1}$ (this was the point of requiring the members of $\mathscr{A}^{\prime}$ to be sequences whose $n$th element has $\leq n+1$ coefficients). Since $\|T\|<\infty$, we have $\lim _{n \rightarrow \infty}\left\|T\left(f_{n}\right)\right\|=0$, so in conclusion, $\lim _{n \rightarrow \infty}\left\|g_{n}\right\|=0$.

\section{Invariants for the Natural Spaces and Very Positive Embeddings}

We will now adapt the Kojman-Shelah method of invariants [6], to the natural spaces and a specific kind of isomorphic embeddings between Banach spaces, which we call very positive embeddings (see Definition 12). From this point on we assume that $\lambda$ is a regular uncountable cardinal.

Definition 10. (1) Suppose that $M$ is a model of size $\lambda$. A filtration of $M$ is a continuous increasing sequence $\left\langle M_{\alpha}: \alpha<\right.$ $\lambda\rangle$ of elementary submodels of $M$, each of size $<\lambda$.

(2) For a regular cardinal $\theta<\lambda$, we use the notation $S_{\theta}^{\lambda}$ for $\{\alpha<\lambda: \operatorname{cf}(\alpha)=\theta\}$.
(3) A club guessing sequence on $S_{\theta}^{\lambda}$ is a sequence $\left\langle C_{\delta}: \delta \epsilon\right.$ $\left.S_{\theta}^{\lambda}\right\rangle$ such that each $C_{\delta}$ is a club in $\delta$, and for every club $E \subseteq \lambda$, there is $\delta$ such that $C_{\delta} \subseteq E$.

Observation 2. Suppose that $\theta>\aleph_{1}$ and there is a club guessing sequence $\left\langle C_{\delta}: \delta \in S_{\theta}^{\lambda}\right\rangle$. Then there is a club guessing sequence $\left\langle D_{\delta}: \delta \in S_{\theta}^{\lambda}\right\rangle$ such that, for all $i<\theta$,

$$
\operatorname{cf}(i) \neq \omega \Longrightarrow \operatorname{cf}\left(\alpha_{i}^{\delta}\right) \neq \omega,
$$

where $\left\langle\alpha_{i}^{\delta}: i\langle\theta\rangle\right.$ is the increasing enumeration of $D_{\delta}$, for each $\delta$.

Proof. First of all notice that by passing to subsets if necessary we can without loss of generality assume that each $C_{\delta}$ has order type $\theta$. Given $\delta$, let $C_{\delta}^{\prime}$ consist of the points of $C_{\delta}$ of cofinality $>\omega$, and let $D_{\delta}$ be the closure of $C_{\delta}^{\prime}$ in $\delta$. Since $\theta>$ $\aleph_{1}$, we have that $C_{\delta}^{\prime}$ is unbounded in $\delta$, so it is clear that $D_{\delta}$ is a club of $\delta$, and since we have $D_{\delta} \subseteq C_{\delta}$, we obtain that the resulting sequence is a club guessing sequence on $S_{\theta}^{\lambda}$. It also follows that $\operatorname{otp}\left(D_{\delta}\right)=\theta$, so the increasing enumeration as claimed exists.

The main definition we need is the definition of the invariant. Let us suppose that $\theta>\aleph_{1}$ is regular and that $\left\langle D_{\delta}: \delta \in S_{\theta}^{\lambda}\right\rangle$ is a club guessing sequence with an increasing enumeration $\left\langle\alpha_{i}^{\delta}: i<\theta\right\rangle$ of $D_{\delta}$, for each $\delta$ and satisfying the requirement (4). This sequence will be fixed throughout. The existence of such a sequence will be discussed at the end of the section, but for the moment let us say that Shelah (see Theorem 15) proved that such a sequence exist in many circumstances, notably for any $\lambda$ regular $\geq \theta^{++}$.

Definition 11. Suppose that $\mathfrak{A}$ is a Boolean algebra of size $\lambda$, $\overline{\mathfrak{A}}=\left\langle\mathfrak{A}_{\alpha}: \alpha<\lambda\right\rangle$ a filtration of $\mathfrak{A}$, that $\delta \in S_{\theta}^{\lambda}$ and that $\bar{f} \epsilon$ $C^{N(\mathfrak{R})} \backslash N\left(\mathfrak{A}_{\delta}\right)$. An ordinal $i \in S_{\neq \omega}^{\theta}$ is an element of the invariant $\operatorname{inv}_{\overline{\mathfrak{Q}}, \delta}(\bar{f})$ if and only if there is $\bar{f}^{\prime} \in C^{N\left(\mathfrak{R}_{\alpha_{i+1}^{\delta}}^{\delta}\right)}$ such that, for every $\bar{g}$ in $C^{N\left(\mathfrak{A}_{\alpha_{i}^{\delta}}\right)}$, we have

$$
0 \leq \lim _{n} g_{n} \leq\left|\lim _{n} f_{n}-\lim _{n} f_{n}^{\prime}\right| \Longrightarrow \bar{g} \in C_{0} .
$$

We will be interested in the kind of embeddings between Banach spaces which will allow us to define appropriate $\phi$ which preserve the invariants; see the Preservation Lemma 13. We have succeeded to do this in the case of a special kind of positive embeddings, as defined in the following definition.

Definition 12. We say that an isomorphic embeddings $T$ : $C(K) \rightarrow C(L)$ is very positive if the following requirements hold:

(i) $g \geq 0 \Rightarrow \mathrm{Tg} \geq 0$ (positivity),

(ii) for every $g \in C(L) \backslash\{0\}$ with $0 \leq g$, there is $h$ with $0 \leq T h \leq g$ and $h \neq 0$,

(iii) if $0 \leq T h \leq T f, h, f \neq 0$, and $f \geq 0$, then there is $s \neq 0$ definable from $h$ with $0 \leq s \leq f$. 
We do not know if very positive embeddings was studied in the literature, but clearly, one kind of embeddings that is very positive is an order preserving onto embeddings. In this case we have Kaplansky's theorem [7] mentioned above, which shows that in the presence of such an embeddings from $C(K)$ onto $C(L)$ we have that $K$ and $L$ are homeomorphic. We show in the example in Section 5 that the analogue is not true for very positive embeddings. In particular the question of the number of pairwise nonisomorphic by very positive embeddings spaces of the form $C(\operatorname{St}(\mathfrak{A}))$ does not reduce to the well-studied and understood question of the number of pairwise nonisomorphic Boolean algebras of a given cardinality (which for any infinite $\kappa$ is always equal to $2^{\kappa}$, see Shelah's [13]).

Let us now make a further assumption on $\lambda$ :

$$
\kappa<\lambda \Longrightarrow \kappa^{\aleph_{0}}<\lambda
$$

Lemma 13 (Preservation Lemma). Let $\mathfrak{A}$ and $\mathfrak{B}$ be Boolean algebras of size $\lambda$ and suppose that $T: C(\operatorname{St}(\mathfrak{A})) \rightarrow C(\operatorname{St}(\mathfrak{B}))$ is a very positive embeddings. Let $\overline{\mathfrak{A}}$ and $\overline{\mathfrak{B}}$ be any filtrations of $\mathfrak{A}$ and $\mathfrak{B}$, respectively, and let $\mathscr{A}$ denote the linear subspace of $C^{N(\mathfrak{A})}$ spanned by the set $\mathscr{A}^{\prime}$ of sequences of functions whose rational coefficients are in $[0,1]$ and that satisfy that the nth coordinate has $\leq n+1$ nonzero coefficients.

If $\phi: \mathscr{A} \rightarrow N(\mathfrak{B})$ is an isomorphic embeddings satisfying that $\lim \phi(\bar{f})=T(\lim (\bar{f}))$ for every $\bar{f} \in \mathscr{A}$, then there is a club $E$ of $\lambda$ such that, for every $\delta$ with $D_{\delta} \subseteq E$ and for every $\bar{f} \in \mathscr{A}^{\prime} \backslash N\left(\mathfrak{A}_{\delta}\right)$ with $0 \leq \lim _{n} f_{n}$ and $\left\|\lim _{n} f_{n}\right\|=1$, we have that

$$
i n v_{\overline{\mathfrak{A}}, \delta}(\bar{f})=i n v_{\overline{\mathfrak{B}}, \delta}(\phi(\bar{f}))
$$

Proof. We may assume that the underlying set of $\mathfrak{A}$ and $\mathfrak{B}$ is the ordinal $\lambda$. Let us define a model $M$ with the universe two disjoint copies of the $\omega$-sequences of the simple functions on $\lambda$ with rational coefficients, interpreted as the elements of $N(\mathfrak{A})$ and $N(\mathfrak{B})$, all the symbols of $N(\mathfrak{A})$ and $N(\mathfrak{B})$ with interpretations induced from these models, and the symbols $\mathscr{A}, \mathscr{A}^{\prime}$, and $\phi$. By the assumption (6), there is a club $E$ of $\lambda$ such that, for every $\delta \in E$ of cofinality not $\omega$, we have that $M$ restricted to the sequences whose ordinal coefficients are $<\delta$ is an elementary submodel of $M$ and that it has universe corresponding to $N\left(\mathfrak{A}_{\delta}\right) \cup N\left(\mathfrak{B}_{\delta}\right)$. Let us denote the latter model by $M\lceil\delta$.

Suppose now that $D_{\delta} \subseteq E$. Choose $\bar{f} \in C^{N(\mathfrak{R})} \cap \mathscr{A}^{\prime} \backslash N\left(\mathfrak{\mathfrak { A }}_{\delta}\right)$ with $0 \leq \lim _{n} f_{n}$ and $\left\|\lim _{n} f_{n}\right\|=1$. Let $f=\lim f_{n}$. By the choice of $\delta$, we have that $\phi(\bar{f}) \in C^{N(\mathfrak{B})} \backslash N\left(\mathfrak{B}_{\delta}\right)$. Suppose first that $i \in \operatorname{inv}_{\overline{\mathfrak{A}}, \delta}(\bar{f})$ and let $\bar{f}^{\prime} \in C^{N\left(\mathfrak{A}_{\alpha_{i+1}^{\delta}}\right)}$ demonstrate this. Let $f^{\prime} \stackrel{\text { def }}{=} \lim f_{n}^{\prime}$, which is well defined as $\bar{f}^{\prime} \in C^{N(\mathfrak{A})}$. Notice that the requirement (5) will hold if we replace $\bar{f}^{\prime}$ by any top-up $\bar{f}^{\prime \prime}$ (see Definition 7) as $0 \leq f \leq 1$, and hence for all $x$, we have $\left|f(x)-f^{\prime \prime}(x)\right| \leq\left|f(x)-f^{\prime}(x)\right|$. Since the topping up procedure is definable in $M \uparrow \alpha_{i+1}^{\delta}$, we may assume that $0 \leq$ $f_{n}^{\prime} \leq 1$ for all $n$ and $0 \leq f^{\prime} \leq 1$. By Lemma 6 applied within $M_{\alpha_{i+1}^{\delta}}$, we can assume that $\bar{f}^{\prime} \in \mathscr{A}$. By the choice of $\phi$, we have that $\lim \phi\left(\bar{f}^{\prime}\right)=T\left(f^{\prime}\right)$ and similarly $0 \leq \lim \phi\left(\bar{f}^{\prime}\right)$. By the fact that $D_{\delta} \subseteq E$ and since $\phi$ is an isomorphism, we have that $\phi\left(\bar{f}^{\prime}\right) \in C^{N\left(\mathfrak{B}_{\alpha_{i+1}^{\delta}}\right)}$. We would like to use $\phi\left(\bar{f}^{\prime}\right)$ to witness that $i \in \operatorname{inv}_{\bar{B}, \delta}(\phi(\bar{f}))$, so let us try. We have already established that $\lim \phi\left(\bar{f}^{\prime}\right)=T\left(f^{\prime}\right)$ and $0 \leq \lim \phi\left(\bar{f}^{\prime}\right)$. It remains to check the property (5) of $\phi\left(\bar{f}^{\prime}\right)$.

Suppose for a contradiction that there is $\bar{g} \in N\left(\mathfrak{B}_{\alpha_{i}^{\delta}}\right)$ such that $0 \leq g \stackrel{\text { def }}{=} \lim _{n} g_{n} \leq\left|T f-T f^{\prime}\right|$ but that $\bar{g} \notin C_{0}$. Applying (ii) we can find $h$ with $0 \leq T h \leq g$ and $h \neq 0$. By Corollary 8 , we can assume that there is $\bar{h} \in \mathscr{A}$ with $h=\lim _{n} h_{n}$, and hence $T h=\lim _{n} \phi(\bar{h})$. Translating the properties guaranteed by (ii) into the terms of $\phi$ and applying the elementarity of $M \uparrow \alpha_{i}^{\delta}$, we can assume that $\bar{h} \in N\left(\mathfrak{A}_{\alpha_{i}^{\delta}}\right)$. Now we apply (iii) to find $s \geq 0, s \neq 0$ definable from $h$ and satisfying $s \leq$ $\left|f-f^{\prime}\right|$. Being definable from $h, s$ has an approximation $\bar{s}$ with $\bar{s}$ definable from $\bar{h}$; hence $\bar{s} \in N\left(\mathfrak{A}_{\alpha_{i}^{\delta}}\right)$. By topping up if necessary as in Lemma 6 and in the above paragraph, we can assume that every element $s_{n}$ in $\bar{s}$ satisfies $s_{n} \geq 0$; therefore $\bar{s}$ contradicts the choice of $\bar{f}^{\prime}$.

Now let us prove the other direction of the desired equality. Let $i \in \operatorname{inv}_{\bar{B}, \delta}(\phi(\bar{f}))$ as exemplified by some $\bar{g} \epsilon$ $N\left(\mathfrak{B}_{\alpha_{i+1}^{\delta}}\right)$. As in the previous paragraphs, we can assume that $0 \leq g \stackrel{\text { def }}{=} \lim _{n} g_{n}$, and hence by (ii) we can assume that for some $f^{\prime}$ which is not 0 we have $0 \leq T f^{\prime} \leq g$ and by the same argument as above we can assume that there is $\bar{f}^{\prime}=$ $\left\langle f_{n}^{\prime}: n<\omega\right\rangle \in N\left(\mathcal{A}_{\alpha_{i+1}^{\delta}}\right) \cap \mathscr{A}$ such that $\lim _{n} f_{n}^{\prime}=f^{\prime}$. Now we claim that $\bar{f}^{\prime}$ exemplifies that $i \in \operatorname{inv}_{\overline{\mathfrak{A}}, \delta}(\bar{f})$. Suppose for a contradiction that $\left\langle h_{n}: n \in \omega\right\rangle \in N\left(\boldsymbol{\mathcal { A }}_{\alpha_{i}^{\delta}}\right) \backslash C_{0}$ and $0 \leq \lim h_{n} \leq\left|\lim f_{n}-\lim f_{n}^{\prime}\right|$. Let $h=\lim h_{n}$. As before, we can assume that $\bar{h} \in \mathscr{A}$ and each $h_{n} \geq 0$. So by the positivity we have $0 \leq T h \leq T\left(\left|f-f^{\prime}\right|\right)=\left|T f-T f^{\prime}\right|$, by the choice of $\phi$ we have that $\phi(\bar{h})=T h$, and by elementarity we have $\phi(\bar{h}) \in N\left(\mathfrak{B}_{\alpha_{i}^{\delta}}\right) \backslash C_{0}$. It follows that $\phi(\bar{h})$ contradicts $i \in \operatorname{inv}_{\overline{\mathfrak{B}, \delta}}(\phi(\bar{f}))$.

The next task is to construct lots of Boolean algebras $\mathfrak{A}$ with different invariants for $N(\mathfrak{A})$ and then to us the Preservation Lemma to show that no fixed $N(\mathfrak{B})$ can embed them all.

Lemma 14 (Construction Lemma). Suppose that $\theta^{+}<\lambda$. Then the club guessing sequence $\left\langle D_{\delta}: \delta \in S_{\theta}^{\lambda}\right\rangle$ can be chosen so that for any $A \subseteq \theta$ which is a closed set of limit ordinals, there is a Boolean algebra $\mathfrak{A}=\mathfrak{A}[A]$, a filtration $\overline{\mathfrak{A}}$ of $\mathfrak{A}$ and a club $E$ of $\lambda$ such that for every $\delta \in E$ there is $\bar{f} \in \mathscr{A}^{\prime} \backslash N\left(\mathfrak{A}_{\delta}\right)$ with $\operatorname{in} v_{\overline{\mathfrak{Q}}, \delta}(\bar{f})=A \cap S_{\neq \omega}^{\theta}$ and $\left\|\lim f_{n}\right\|=1$.

The proof of this lemma is presented in Section 6. The following theorem of Shelah will be used in the proof of the Construction Lemma as well as in the proof of Theorem 16. 
Theorem 15 (Shelah, [14, Claim 1.4]). Let $\theta<\lambda$ be two regular cardinals with $\theta^{+}<\lambda$. Then there is a stationary set $S \subseteq S_{\theta}^{\lambda}$ and sequences $\left\langle c_{\delta}: \delta \in S\right\rangle,\left\langle\mathscr{P}_{\alpha}: \alpha<\lambda\right\rangle$ such that

(i) $\operatorname{otp}\left(c_{\delta}\right)=\theta$ and $\sup \left(c_{\delta}\right)=\delta$;

(ii) for every club $E$ of $\lambda$, there is $\delta \in S$ with $c_{\delta} \subseteq E$;

(iii) $\mathscr{P}_{\alpha} \subseteq \mathscr{P}(\alpha)$ and $\left|\mathscr{P}_{\alpha}\right|<\lambda$;

(iv) if $\alpha$ is a nonaccumulation point of $c_{\delta}$, then $c_{\delta} \cap \alpha \in$ $\bigcup_{\alpha^{\prime}<\alpha} \mathscr{P}_{\alpha}^{\prime}$;

(v) the nonaccumulation points of every $c_{\delta}$ are successor ordinals.

Claim 1.4. in [14] does not state property (c) explicitly, but it follows from the first line of the proof of that Claim.

Now we present the main theorem of the paper.

Theorem 16. Suppose that $\theta$ and $\lambda$ are two regular cardinals with $\aleph_{2} \leq \theta<\theta^{+}<\lambda<2^{\theta}$ and that $(\forall \kappa<\lambda) \kappa^{\aleph_{0}}<\lambda$.

Then

(1) the minimal number of spaces of the form $C(S t(\mathfrak{A}))$ of density $\lambda$ needed to embed all Banach spaces of the form $C(S t(\mathfrak{B}))$ of density $\lambda$ very positively is $2^{\theta}$. In particular there is no very-positively universal space $C(S t(\mathfrak{A}))$ of density $\lambda$;

(2) there are at least $c f\left(2^{\theta}\right)$ pairwise non-very positively isomorphic Banach spaces $C(K)$ of density $\lambda$.

Proof. Fix sequences $\left\langle c_{\delta}: \delta \in S \subseteq S_{\theta}^{\lambda}\right\rangle$ and $\left\langle\mathscr{P}_{\alpha}: \alpha<\lambda\right\rangle$ as guaranteed by Theorem 15 . Notice that $\left\langle c_{\delta}: \delta \in S\right\rangle$ satisfies that, with $\left\langle\alpha_{i}^{\delta}: i<\theta\right\rangle$ being the increasing enumeration of $c_{\delta}$, we have that $\operatorname{cf}(i) \neq \omega \Rightarrow \operatorname{cf}\left(\alpha_{i}^{\delta}\right) \neq \omega$. Hence letting $D_{\delta}=c_{\delta}$ for $\delta \in S$ and $D_{\delta}$ an arbitrary club of $\delta$ of order type $\theta$ satisfying $\operatorname{cf}(i) \neq \omega \Rightarrow \operatorname{cf}\left(\alpha_{i}^{\delta}\right) \neq \omega$ for $\delta \in S_{\theta}^{\lambda} \backslash S$, the sequence can be used in the context of the Preservation Lemma 13. It can also be used in the context of the Construction Lemma 14. Let us therefore find the Boolean algebras $\mathfrak{A}[A]$ as described in the statement of the Construction Lemma. Notice that there are $2^{\theta}$ many different choices for $A \cap S_{\neq \omega}^{\theta}$.

(1) Suppose for a contradiction that there is a family $\left\{C\left(\operatorname{St}\left(\mathfrak{A}_{\alpha}\right)\right): \alpha<\alpha^{*}\right\}$ for some $\alpha^{*}<2^{\theta}$ for some algebras $\mathfrak{A}_{\alpha}$ of size $\lambda$ which is very positively universal for all $C(\operatorname{St}(\mathfrak{A}))$ for Boolean algebras $\mathfrak{A}$ of size $\lambda$. Notice that the assumptions we have made on $\lambda$ imply that $\lambda^{\aleph_{0}}=\lambda$, so the size of each $N\left(\mathfrak{A}_{\alpha}\right)$ is $\lambda$. Let $\mathscr{F}$ be the family of all subsets of $\theta$ that appear as invariants of elements of $\bigcup_{\alpha<\alpha^{*}} N\left(\mathfrak{A}_{\alpha}\right)$; hence the size of $\mathscr{F}$ is $<2^{\theta}$ (since we have assumed $\lambda<2^{\theta}$ ), and in particular there is $A \subseteq \theta$ a closed set of limit ordinals such that $A \cap S_{\neq \omega}^{\theta} \notin \mathscr{F}$. Let $\mathfrak{A}=\mathfrak{A}[A]$. Suppose that $T$ is a very positive embeddings of $C(\operatorname{St}(\mathfrak{A}))$ into some $C\left(\operatorname{St}\left(\mathfrak{A}_{\alpha}\right)\right)$, and let $\phi$ be an embeddings of $N(\mathfrak{A})$ into $N\left(\mathfrak{A}_{\alpha}\right)$ satisfying that for every $\bar{f} \in \mathscr{A} \cap C^{N(\mathfrak{R})}$, we have $\phi(\bar{f})=T(f)$, which exists by Theorem 9. Let $E_{0}$ be a club of $\lambda$ as guaranteed by the Preservation Lemma, let $E_{1}$ be a club of $\lambda$ as guaranteed by the Construction Lemma, let $E=E_{0} \cap E_{1}$, and suppose that $\delta$ is such that $D_{\delta} \subseteq E$. Then by the choice of $E_{0}$ there is $\bar{f}$ in $N(\mathfrak{A})$ whose invariant is $A \cap S_{\neq \omega}^{\theta}$, but then $\phi(\bar{f})$ also has invariant $A \cap S_{\neq \omega}^{\theta}$, by the choice of $E_{1}$, and hence we have a contradiction with the choice of $A$.

(2) Consider the family $\mathscr{G}=\{C(\operatorname{St}(\mathfrak{A}[A]))$ : $A$ a closed set of limit ordinals in $\theta\}$. By the argument in 1 for every $A$, the set $\{B$ a closed set of limit ordinals in $\theta$ : $C(\operatorname{St}(\mathfrak{A}[B]))$ that embeds very positively into $C(\operatorname{St}(\mathfrak{H}[A]))\}$ has size $<2^{\theta}$, so clearly every $C(\operatorname{St}(\mathfrak{A}[A]))$ is very positively isomorphic with $<2^{\theta}$ many $C(\operatorname{St}(\mathfrak{A}[B]))$. Hence we can choose $\operatorname{cf}\left(2^{\theta}\right)$ pairwise non-very positively isomorphic elements of $\mathscr{G}$ by a simple induction.

\section{Examples}

5.1. Cardinal Arithmetic. An example of circumstances when Theorem 16 applies is when

$$
\theta=\aleph_{2}, \quad \lambda=\aleph_{4}, \quad 2^{\aleph_{0}}=\aleph_{1} \quad \text { but } 2^{\aleph_{1}} \geq \aleph_{5} .
$$

5.2. Very Positive Embeddings onto Do Not Give Rise to Homeomorphism. We give an example of two 0 -dimensional spaces $K$ and $L$ which are not homeomorphic; yet they admit a very-positive isomorphism onto. The example itself was constructed by Plebanek in [15, Example 5.3], when considering positive onto isomorphisms.

Let $K$ consist of two disjoint convergent sequences $\left\langle x_{n}\right.$ : $n<\omega\rangle$ with $\lim _{n} x_{n}=x$ and $\left\langle y_{n}: n<\omega\right\rangle$ with $\lim _{n} y_{n}=y \neq x$, and let $L$ consist of a single convergent sequence $\left\langle z_{n}: n<\omega\right\rangle$ with $\lim _{n} z_{n}=z$. Define $T: C(K) \rightarrow C(L)$ by letting for all $n$

$$
\begin{aligned}
\operatorname{Tf}\left(z_{0}\right) & =f(y), \\
\operatorname{Tf}\left(z_{2 n-1}\right) & =\frac{f\left(x_{n}\right)+f(y)}{2}, \\
\operatorname{Tf}\left(z_{2 n+2}\right) & =\frac{f(x)+f\left(y_{n}\right)}{2} .
\end{aligned}
$$

Plebanek shows that $T$ is a positive isomorphism onto $C(L)$ and moreover he calculates the inverse $S=T^{-1}$ which is given by

$$
\begin{gathered}
\operatorname{Sh}(y)=h\left(z_{0}\right), \quad \operatorname{Sh}(x)=2 h(z)-h\left(z_{0}\right), \\
\operatorname{Sh}\left(x_{n}\right)=2 h\left(z_{2 n-1}\right)-h\left(z_{0}\right), \\
\operatorname{Sh}\left(y_{n}\right)=2 h\left(z_{2 n}\right)-2 h(z)+h\left(z_{0}\right) \\
\text { for } n \geq 1 .
\end{gathered}
$$

We will show that $T$ is a very positive embeddings. Considering property (ii) of Definition 12, suppose that $g \in C(L) \backslash\{0\}$ with $0 \leq g$; we need to find $h$ with $0 \leq T h \leq g$ and $h \neq 0$. Since $T$ is onto, there is $h$ such that $T h=g$ and since $T$ is an isomorphism and $g \neq 0$, we also have $h \neq 0$.

For the property (iii), we will have an existential proof of the existence of the $s$ as required, given $h$ and $f$ as in the assumptions. First let us deal with the case that $h \geq 0$. Let $\delta$ be the family of all non-negative functions in $C(K)$ for which there is exactly one point with non-zero value, and on that point the value is equal to that of $h$. Each element of $\mathcal{S}$ is 
clearly definable from $h$. We claim that some $s \in \mathcal{S}$ can be chosen to demonstrate (iii). Namely, since we do not have $T f \leq T h$, we cannot have $f \leq h$ by positivity. Hence there is some value $w$ with $h(w)<f(w)$, and by the continuity of the functions $h$ and $f$, there must be some such $w \in\left\{x_{n}, y_{n}\right.$ : $n \in \omega\}$. Then letting $s(w)=h(w)$ and $s(v)=0$ for $v \neq w$ gives a function in $\mathcal{S}$, and we have $0 \leq s \leq f$ and $0 \neq s$. Let us now deal with the general case.

Since $0 \leq T h \leq T f$, it follows that $h(y) \geq 0$. Suppose first that $h(y)>0$; hence certainly $f(y)>0$. Let $\varepsilon>0$ be such that $\varepsilon \cdot h(y)<f(y)$ (so we can take $\varepsilon=1$ if $h(y)<f(y)$ and $\varepsilon=1 / 2$ if $h(y)=f(y))$. We are going to define a function $s_{n} \in C(K)$ for $n<\omega$ by letting $s_{n}(y)=\varepsilon h(y), s_{n}\left(y_{m}\right)=\varepsilon h\left(y_{m}\right)$ for $m \geq n$ and $s_{n}=0$ otherwise. Since $h$ is continuous and $h(y)>0$, we have, that for large enough $n, h\left(y_{n}\right) \geq 0$, and hence $s_{n} \geq 0$ and $s_{n} \neq 0$. Since $\varepsilon \cdot h(y)<f(y)$ and both $h$ and $f$ are continuous, we must have that, for large enough $n$, $\varepsilon h\left(y_{n}\right)<f\left(y_{n}\right)$, and therefore for large enough $n$ we have $s_{n} \leq f$. So, some $s_{n}$ will work to exemplify (iii).

Suppose now that $h(y)=0$. If $h(x)>0$, by continuity $h\left(x_{n}\right)$ is eventually $>0$. If $f(x)>0$, we can choose $\varepsilon$ as in the previous paragraph, so we are done by a similar argument. Otherwise we have that $f(x)=0$ so by looking at $T h\left(z_{2 n+2}\right)$ and $T f\left(z_{2 n+2}\right)$, we obtain $h(x) \leq f(y)$. Also we have that $h\left(x_{n}\right)$ is eventually $>0$. Choose $\varepsilon>0$ such that $\varepsilon \cdot h(x)<f(y)$ and define $s_{n}$ by letting $\varepsilon \cdot s_{n}(y)=h(x)$ and for $m \geq n$, $s_{n}\left(y_{m}\right)=\varepsilon \cdot h\left(x_{m}\right)$, we see that for large enough $n$, we have that $0 \leq s_{n} \leq f$.

If $h(x)<0$ then eventually $T h\left(z_{2 n+2}\right)<0$, which is a contradiction and so $h(x) \geq 0$. If $h(x)=0$ then we conclude from the definition of Th that, for every $n, h\left(x_{n}\right)=2 \cdot T h\left(z_{2 n-1}\right) \geq$ 0 and similarly $h\left(y_{n}\right)=2 \cdot T h\left(z_{2 n+2}\right) \geq 0$, therefore $h \geq 0$, and we can use the very first argument.

\subsection{Specific Very Positive Embeddings}

\section{Theorem 17. Theorem 16(1) implies Theorem 1.}

Proof. We just need to show that the assumptions of Theorem 1 imply those of Theorem 16(1). The cardinal arithmetic assumption and the requirement of positivity are the same in both theorems, so we proceed to show that any $T$ as in the assumptions of Theorem 1 satisfies the requirements (ii) and (iii) of the definition of very positivity. Requirement (ii) follows easily by the surjectivity of $T$. Finally (iii), letting $a, b$ be as given by (iii) in Theorem 1 and $s=a \cdot h+b$ suffices for (iii) of the very positivity.

5.4. Not Every Positive onto Embeddings Satisfies the Requirements of Theorem 1. Note that it is a consequence of the assumptions of Theorem 1 that if $T h \leq T f$, then for every $x$, $f(x)=0 \Rightarrow h(x) \leq-b / a$.

\section{Proof of the Construction Lemma}

We present a proof of Lemma 14. Let $S \subseteq S_{\theta}^{\lambda}$ and sequences $\left\langle c_{\delta}: \delta \in S\right\rangle,\left\langle\mathscr{P}_{\alpha}: \alpha<\lambda\right\rangle$ be as in the statement of Theorem 15, while $\left\langle D_{\delta}: \delta \in S_{\theta}^{\lambda}\right\rangle$ is such that $D_{\delta}=c_{\delta}$ for $\delta \in S$. For all the definitions of invariants we use here, the value of the invariant is the same with respect to $\left\langle c_{\delta}: \delta \in S\right\rangle$ as it is with respect to $\left\langle D_{\delta}: \delta \in S_{\theta}^{\lambda}\right\rangle$, so we will not make a difference between the two. We start with a construction lemma for a certain family of linear orders, as obtained by Kojman and Shelah in [6]. Let us give their definition of the invariants of linear orders.

Definition 18. Suppose that $L$ is a linear order with the universe $\lambda$ and $\mathbb{Q}=\left\langle L_{\delta}: \delta\langle\lambda\rangle\right.$ is a filtration of $L$. Then for every $\delta \in S$ such that the universe of $L_{\delta}$ is $\delta$, we define

$$
\begin{aligned}
\operatorname{inv}_{\mathfrak{Q}, \delta}(\delta) \stackrel{\text { def }}{=}\{i & <\theta:\left(\exists \delta^{\prime} \in\left(\alpha_{i}^{\delta}, \alpha_{i+1}^{\delta}\right]\right)\left(\forall x \in L_{\alpha_{i}^{\delta}}\right) x \leq_{L} \delta \\
& \left.\Longleftrightarrow x \leq_{L} \delta^{\prime}\right\} .
\end{aligned}
$$

Lemma 3.7 in [6] proves that, under the assumptions we have stated, for every closed set $A$ of limit ordinals in $\theta$, there is a linear order $L[A]$ with universe $\lambda$ and a filtration $\mathfrak{Q}[A]=$ $\left\langle L_{\delta}[A]: \delta<\lambda\right\rangle$ of $L[A]$ such that for every $\delta \in S$ with $L_{\delta}[A]=\delta$ we have $\operatorname{inv}_{\mathfrak{Q}[A], \delta}(\delta)=A$ (Lemma 3.7 in [6] also states the assumption $2^{\theta}<\lambda$, but this assumption is not used in the proof of the lemma, only in the proof of the final result).

The idea of our proof is to transform the Kojman-Shelah construction first into a construction of a family of Boolean algebras of size $\lambda$ and then to use these Boolean algebras to define natural spaces of functions with appropriate invariants.

Definition 19. Suppose that $\mathfrak{A}$ is a Boolean algebra with the set of generators $\left\{a_{\alpha}: \alpha<\lambda\right\}$ and $\overline{\mathfrak{A}}=\left\langle\boldsymbol{\mathcal { P }}_{\delta}: \delta<\lambda\right\rangle$ is a filtration of $\mathfrak{A}$, while $\delta \in S$ is such that $\mathfrak{A}_{\delta}$ is generated by $\left\{a_{\alpha}: \alpha<\delta\right\}$. We define

$$
\begin{gathered}
\operatorname{inv}_{\overline{\mathfrak{Q}}, \delta}\left(a_{\delta}\right) \stackrel{\text { def }}{=}\left\{i<\theta:\left(\exists \delta^{\prime} \in\left(\alpha_{i}^{\delta}, \alpha_{i+1}^{\delta}\right]\right)\left(\forall \alpha<\alpha_{i}^{\delta}\right)\right. \\
a_{\alpha} \cap a_{\delta}=a_{\alpha} \cap a_{\delta}^{\prime} \bmod \mathfrak{\mathfrak { A }}_{\alpha_{i}^{\delta}}, \\
\left.a_{\alpha}^{c} \cap a_{\delta}=a_{\alpha}^{c} \cap a_{\delta}^{\prime} \bmod \mathfrak{\mathfrak { A }}_{\alpha_{i}^{\delta}}\right\},
\end{gathered}
$$

where $a=b \bmod \mathfrak{A}_{\alpha_{i}^{\delta}}$ means that for any element $w$ of $\mathfrak{A}_{\alpha_{i}^{\delta}}$ we have $w \leq a$ if and only if $w \leq b$.

Definition 20. Suppose that $L$ is a linear order with universe $\lambda$. We define a Boolean algebra $\mathfrak{A}[L]$ as being generated by $\left\{a_{\alpha}: \alpha<\lambda\right\}$ freely except for the equations

$$
a_{\delta} \leq a_{\varepsilon} \Longleftrightarrow \delta \leq_{L} \varepsilon
$$

Since the equations in (13) are finitely consistent with the axioms of a Boolean algebra, it follows from the compactness theorem that the algebra $\mathfrak{A}[L]$ is well defined. Now we will see a translation between the calculation of the invariants of the linear orders and the associated Boolean algebras.

Sublemma 14.1. Let $L$ be a linear order on $\lambda$, and let $\mathfrak{A}[L]$ be the algebra associated to $L$ as per Definition 20. Let $\bar{L}$ and $\overline{\mathfrak{A}}$ be 
any filtrations of $L$ and $\mathfrak{A}[L]$, respectively. Then there is a club $E$ such that for every $\delta \in S \cap E$ one has

$$
i n v_{\bar{L}, \delta}(\delta)=i n v_{\overline{\mathfrak{A}}, \delta}\left(a_{\delta}\right)
$$

and moreover, for any $i \in i n v_{\bar{L}, \delta}(\delta)$, this is exemplified by $\delta^{\prime}$ if and only if $i \in \operatorname{inv} v_{\overline{\mathfrak{Q}}, \delta}\left(a_{\delta}\right)$ is exemplified by $a_{\delta^{\prime}}$.

Proof. Let $E$ be the club of $\delta$ such that the universe of $L_{\delta}$ is $\delta, L_{\delta}$ is an elementary submodel of $L$, and $\mathfrak{A}[L]_{\delta}$ is generated by $\left\{a_{\alpha}: \alpha<\delta\right\}$ and is an elementary submodel of $\mathfrak{A}$. Suppose that $\delta \in E \cap S$.

First suppose that $i \in \operatorname{inv}_{\mathcal{\Omega}, \delta}(\delta)$ as exemplified by $\delta^{\prime}$. Let $\alpha<\alpha_{i}^{\delta}$, we need to prove $a_{\alpha} \cap a_{\delta}=a_{\alpha} \cap a_{\delta}^{\prime} \bmod \mathfrak{A}_{\alpha_{i}^{\delta}}$ and $a_{\alpha}^{c} \cap$ $a_{\delta}=a_{\alpha}^{c} \cap a_{\delta}^{\prime} \bmod \mathfrak{A}_{\alpha_{i}^{\delta}}$.

Case $1\left(\alpha<_{L} \delta\right)$. Hence by the choice of $\delta^{\prime}$, we have $\alpha<_{L} \delta^{\prime}$ and $a_{\alpha} \leq a_{\delta}, a_{\alpha} \leq a_{\delta}^{\prime}$. Therefore $a_{\alpha} \cap a_{\delta}=a_{\alpha} \cap a_{\delta}^{\prime}=a_{\alpha}$.

Suppose that $z>0$ is in $\mathfrak{\mathcal { A }}_{\alpha_{i}^{\delta}}$ and satisfies $z \leq a_{\alpha}^{c} \cap a_{\delta}$. By the Disjunctive Normal Form for Boolean algebras, we can assume that $z=\bigvee_{i \leq n} \bigwedge_{j \leq k_{i}} a_{\beta(i, j)}^{l(i, j)}$ for some $l(i, j) \in\{0,1\}$ and $\beta(i, j)<\alpha_{i}^{\delta}$. It suffices to prove that for every $i$ we have $\bigwedge_{j \leq k_{i}} a_{\beta(i, j)}^{l(i, j)} \leq a_{\alpha}^{c} \cap a_{\delta}^{\prime}$. Fix an $i$ and without loss of generality assume that $\bigwedge_{j \leq k_{i}} a_{\beta(i, j)}^{l(i, j)}>0$, as otherwise the conclusion is trivial.

Let $A_{l}=\left\{j \leq k_{i}: \beta(i, j)=l\right\}$, for $l \in\{0,1\}$. For simplicity assume that both of these sets are nonempty, as otherwise the proof is easier. Let $\beta_{1}$ be the $L$-minimal element of $A_{1}$; hence $\bigwedge_{j \in A_{1}} a_{\beta(i, j)}^{l(i, j)}=a_{\beta_{1}}$. Let $\beta_{0}$ be the $L$-maximal element of $A_{0}$; hence $\bigwedge_{j \in A_{0}} a_{\beta(i, j)}^{c}=\left(\bigvee_{j \in A_{0}} a_{\beta(i, j)}\right)^{c}=a_{\beta_{0}}^{c}$. In conclusion, $\bigwedge_{j \leq k_{i}} a_{\beta(i, j)}^{l(i, j)}=a_{\beta_{0}}^{c} \cap a_{\beta_{1}}$. Since we have assumed that $\bigwedge_{j \leq k_{i}} a_{\beta(i, j)}^{l(i, j)}>0$, we cannot have $a_{\beta_{1}} \leq a_{\beta_{0}}$, equivalently $\beta_{1} \leq_{L} \beta_{0}$. Hence we have $\beta_{0}<_{L} \beta_{1}$. Similarly, since $a_{\beta_{1}} \cap a_{\alpha}^{c}>0$ we can conclude that $\alpha<_{L} \beta_{1}$. Finally, if we had $\beta_{0}>_{L} \delta$, then we would obtain $a_{\beta_{0}}^{c} \leq a_{\delta}^{c}$, in contradiction with $0<a_{\beta_{0}^{c}} \cap$ $a_{\beta_{1}} \leq a_{\delta}$, and therefore $\beta_{0}<_{L} \delta$.

Suppose now that $\delta<_{L} \beta_{1}$. Therefore $a_{\beta_{1}} \cap a_{\delta}^{c} \neq 0$. On the other hand, $a_{\beta_{0}}^{c} \cap a_{\beta_{1}} \cap a_{\delta}^{c} \leq a_{\delta} \cap a_{\alpha^{c}} \cap a_{\delta^{c}}=0$, and hence we must have $a_{\beta_{0}} \cap a_{\beta_{1}} \cap a_{\delta}^{c}>0$, which, taking into account $\beta_{0}<_{L} \beta_{1}$, gives that $a_{\beta_{0}} \cap a_{\delta}^{c}>0$, and hence $\delta<_{L} \beta_{0}$, a contradiction. Hence we have $\beta_{1}<_{L} \delta$. By the choice of $\delta^{\prime}$, we have $\beta_{1}<_{L} \delta^{\prime}$, and hence $a_{\beta_{1}} \leq a_{\delta^{\prime}}$ and in particular $a_{\beta_{0}}^{c} \cap a_{\beta_{1}} \leq a_{\delta^{\prime}}$, as required. Since the roles of $\delta$ and $\delta^{\prime}$ in this proof were symmetric, we can prove in the same way that for any $z>0$ in $\mathfrak{U}_{\alpha_{i}^{\delta}}$ which satisfies $z \leq a_{\alpha}^{c} \cap a_{\delta}^{\prime}$ we also have $z \leq a_{\alpha}^{c} \cap a_{\delta}$.

Case $2\left(\alpha>_{L} \delta\right.$, so $\alpha>_{L} \delta^{\prime}$ by the choice of $\left.\delta^{\prime}\right)$. We have $a_{\delta}^{c} \geq$ $a_{\alpha}^{c}$, so $a_{\alpha}^{c} \cap a_{\delta}=0$, and similarly $a_{\alpha}^{c} \cap a_{\delta}^{\prime}=0$. We also have $a_{\alpha} \cap a_{\delta}=a_{\delta}$ and similarly for $\delta^{\prime}$; hence we need to prove that $a_{\delta}=a_{\delta^{\prime}} \bmod \mathfrak{A}_{\alpha_{i}^{\delta}}$. As in Case 1, it suffices to show that, for every $\beta_{0}, \beta_{1}<\alpha_{i}^{\delta}$ with $0<a_{\beta_{0}}^{c} \cap a_{\beta_{1}}<a_{\delta}$, we have $a_{\beta_{0}}^{c} \cap$ $a_{\beta_{1}} \leq a_{\delta}^{\prime}$ (the equality cannot occur), and vice versa. Let us start with the forward direction. As before, from $0<a_{\beta_{0}}^{c} \cap a_{\beta_{1}}$, we conclude $\beta_{0}<_{L} \beta_{1}$. Also, if $\beta_{0}>_{L} \delta$, then we have $a_{\beta_{0}}^{c} \leq a_{\delta}^{c}$, contradicting that $a_{\beta_{0}}^{c} \cap a_{\delta}>0$. Hence $\beta_{0}<_{L} \delta$.

If $\beta_{1}<_{L} \delta$, then $\beta_{1}<_{L} \delta^{\prime}$, so $a_{\beta_{1}} \leq a_{\delta}^{\prime}$, and hence $a_{\beta_{0}}^{c} \cap a_{\beta_{1}} \leq$ $a_{\delta}^{\prime}$, as required. So assume that $\delta<_{L} \beta_{1}$. Hence $a_{\beta_{1}}>a_{\delta}$ and so $a_{\beta_{0}}^{c} \cap a_{\beta_{1}}>a_{\beta_{0}}^{c} \cap a_{\delta} \geq a_{\beta_{0}}^{c} \cap a_{\beta_{1}}$, a contradiction. This finishes the proof of the forward direction, and the other direction follows from the symmetry of the roles of $\delta$ and $\delta^{\prime}$ in the proof.

Now suppose that $i \in \operatorname{inv}_{\overline{\mathfrak{Q}}, \delta}\left(a_{\delta}\right)$ as exemplified by $a_{\delta}^{\prime}$. Let $\alpha<\alpha_{i}^{\delta}$; we need to prove $\alpha<_{L} \delta \Leftrightarrow \alpha<_{L} \delta^{\prime}$. If $\alpha<_{L} \delta$, then $a_{\alpha}<a_{\delta}$, hence $a_{\alpha}<a_{\delta^{\prime}}$ by the assumption, and hence $\alpha<{ }_{L} \delta^{\prime}$ by the definition of $\mathfrak{A}[L]$. The other direction follows by symmetry.

The next step is going from the invariants of Boolean algebras to the invariants of natural spaces.

Sublemma 14.2. Let $\mathfrak{A}[L]$ be one of the algebras described in the above, and let $\overline{\mathfrak{A}}$ be its filtration. Then there is a club $E$ of $\lambda$ such that, for every $\delta \in S$ with $D_{\delta} \subseteq E$, one has that

$$
\operatorname{in} v_{\overline{\mathfrak{A}}, \delta}\left(\chi_{\left[a_{\delta}\right]}\right)=i n v_{\overline{\mathfrak{A}}, \delta}\left(a_{\delta}\right)
$$

and moreover, for any $i \in \operatorname{in} v_{\overline{\mathfrak{A}}, \delta}\left(\chi_{\left[a_{\delta}\right]}\right)$, this is exemplified by $\chi_{\left[a_{\delta}^{\prime}\right]}$ if and only if $i \in \operatorname{inv} \overline{\mathfrak{A}, \delta}_{\delta}\left(a_{\delta}\right)$ is exemplified by $a_{\delta^{\prime}}$. Here, the invariant on the left refers to the invariant in the natural space $N(\mathfrak{U})$ and the invariant on the right to the invariant in the algebra $\mathfrak{A}$. The notation $\chi_{[a]}$ is used for the sequence $\left\langle\chi_{[a]}, \chi_{[a]}, \chi_{[a]}, \ldots\right\rangle$ in $C^{N(\mathfrak{A})}$

Proof. Let $\mathfrak{M}^{*}$ be a model consisting of $L, \mathfrak{A}$, two disjoint copies of the $\omega$-sequences of the simple functions on $\lambda$ with rational coefficients, interpreted as the elements of $N(\mathfrak{A})$ and all the symbols of $N(\boldsymbol{\Re})$ with induced interpretations induced from these models. Recall the assumption that for all $\kappa<\lambda$, we have $\kappa^{\aleph_{0}}<\lambda$ and notice that it implies that there is a club $E_{0}$ of $\lambda$ such that, for every $\delta \in E_{0}$ of cofinality $>\aleph_{0}$, the model $\mathfrak{M}^{*}\left\lceil\delta\right.$ is $\aleph_{1}$-saturated in $\mathfrak{M}^{*}$; that is, it realizes all the types with countably many parameters in $\mathfrak{M}^{*}\lceil\delta$ which are realized in $\mathfrak{M}^{*}$. Let $\bar{L}$ be any filtration of $L$, let $E \subseteq E_{0}$ be a club witnessing Sublemma 14.1, and let $\delta \in S$ be such that $D_{\delta} \subseteq E$.

Suppose $i \in \operatorname{inv}_{\overline{\mathfrak{A}}, \delta}\left(a_{\delta}\right)$ as exemplified by $a_{\delta^{\prime}}$ but $\bar{g} \geq 0$ with $\bar{g} \in C^{N\left(\mathfrak{A}_{\alpha_{i}^{\delta}}\right)} \backslash C_{0}$ and the limit $g$ of $\bar{g}$ satisfies $g \leq$ $\left|\chi_{\left[a_{\delta}\right]}-\chi_{\left[a_{\delta}^{\prime}\right]}\right|=\chi_{\left[a_{\delta} \Delta a_{\delta^{\prime}}\right]}$. By topping up if necessary (see Definition 7), we may assume that each $g_{n} \geq 0$, and by throwing away unnecessary elements of $\bar{g}$, we may assume that every $g_{n} \neq 0$. We can then assume that for each $n$ there are pairwise disjoint $\left\{b_{0}^{n}, \ldots, b_{n}^{k_{n}}\right\} \in \mathcal{\mathfrak { A }}_{\alpha_{i}^{\delta}}$ and $q_{i}^{n}\left(i \leq k_{n}\right) \in$ $\mathbb{Q}^{+}$such that $g_{n}=\sum_{i \leq k_{n}} q_{i}^{n} \chi_{\left[b_{i}^{n}\right]}$. Since $\left\|g_{n}-\chi_{\left[a_{\delta} \Delta a_{\delta^{\prime}}\right]}\right\| \rightarrow$ 0 , there has to be a $\left[b_{i}^{n}\right]$ with a nonempty intersection with $\left[a_{\delta} \Delta a_{\delta^{\prime}}\right]$. By applying the Disjunctive Normal Form, we can assume that $b_{i}^{n}=\bigvee_{j \leq m} \bigwedge_{o \leq o_{m}} a_{\beta(m, o)}^{l(m, o)}$ for some $l(m, o) \in\{0,1\}$ and $\beta(m, o)<\alpha_{i}^{\delta}$. Therefore there is $j \leq m$ such that $\bigwedge_{o \leq o_{m}}\left[a_{\beta(m, o)}^{l(m, o)}\right] \cap\left[a_{\delta} \Delta a_{\delta^{\prime}}\right] \neq \emptyset$. Then we have that $\bigwedge_{o \leq o_{m}}\left[a_{\beta(m, o)}^{l(m, o)}\right] \cap\left[a_{\delta}\right] \neq \bigwedge_{o \leq o_{m}}\left[a_{\beta(m, o)}^{l(m, o)}\right] \cap\left[a_{\delta}^{\prime}\right]$, and hence there 
has to be $o \leq p_{m}$ such that $\left[a_{\beta(m, o)}^{l(m, o)}\right] \cap\left[a_{\delta}\right] \neq\left[a_{\beta(m, o)}^{l(m, o)}\right] \cap\left[a_{\delta}^{\prime}\right]$. It follows that $\left[a_{\beta(m, o)}^{1-l(m, o)}\right] \cap\left[a_{\delta}\right] \neq\left[a_{\beta(m, o)}^{1-l(m, o)}\right] \cap\left[a_{\delta}^{\prime}\right]$. Let $\beta=$ $\beta(m, 0)$. From the choice of $E$, using Sublemma 14.1, we have that for $R \in\left\{<_{L},>_{L}\right\}, \beta R \delta$ if and only if $\beta R \delta^{\prime}$. We go through a case analysis like in the proof of Sublemma 14.1. If $\beta<_{L} \delta$, then we have $\beta<_{L} \delta^{\prime}$, so $\left[a_{\beta}\right] \cap\left[a_{\delta}\right]=\left[a_{\beta}\right]=\left[a_{\beta}\right] \cap\left[a_{\delta^{\prime}}\right]$, a contradiction. If $\beta>_{L} \delta$, then $\left[a_{\beta}^{c}\right] \cap\left[a_{\delta}\right]=\emptyset=\left[a_{\beta}^{c}\right] \cap\left[a_{\delta^{\prime}}\right]$, a contradiction. Therefore, $i \in \operatorname{inv}_{\overline{\mathfrak{A}}, \delta}\left(\chi_{\left[a_{\delta}\right]}\right)$.

Claim 1. Suppose that $i \in \operatorname{inv}_{\overline{\mathfrak{A}}, \delta}\left(\chi_{\left[a_{\delta}\right]}\right)$ as exemplified by some $\bar{f}$. Without loss of generality, we can assume that $\bar{f}=\chi_{\left[a_{\delta}^{\prime}\right]}$ for some $\delta^{\prime}$.

Proof of the Claim. First let us notice that if $f=\lim _{n} f_{n}$, then for $f^{\prime}=\min \{f, 1\}$, we have $\left|\chi_{\left[a_{\delta}\right]}-f^{\prime}\right| \leq\left|\chi_{\left[a_{\delta}\right]}-f\right|$, so we can without loss of generality assume that $f \leq 1$. Similarly we can assume that $f \geq 0$, and then by applying a similar logic, we can also assume that $0 \leq f_{n} \leq 1$ for all $n$ and that $f_{n} \neq 0$ for all $n$. Each $f_{n}$ is a simple function with rational coefficients defined on (without loss of generality) disjoint basic clopen sets of the form $\left[a_{\beta}^{l_{\beta}}\right]$ where $\beta<\alpha_{i+1}^{\delta}$ and $l_{\beta}<2$. Let $\left\{\beta_{n}: n<\right.$ $\omega\}$ enumerate all the relevant $\beta$. For each $n$ and $R \in\left\{<_{L},>_{L}\right\}$, let $j_{R}^{n}$ be the truth value of " $a_{\beta_{n}} R a_{\delta}$ ". Consider the following sentence with parameters $\bar{f}, \alpha_{i}^{\delta}$ and the elements of $\left\{\beta_{n}: n<\right.$ $\omega\}$; there is $\beta$ such that

(i) for all $\bar{g} \in N\left(\mathfrak{\mathcal { Y }}_{\alpha_{i}^{\delta}}\right)$ if $0 \leq \lim _{n} g_{n} \leq\left|\lim f_{n}-\chi_{\left[a_{\beta}\right]}\right|$, we have $\bar{g} \in C^{0}$;

(ii) for all $n$ and $R \in\left\{<_{L},>_{L}\right\}$, we have $a_{\beta_{n}} R a_{\beta}$ if and only if $j_{R}^{n}=1$.

This sentence is true as exemplified by $\delta$, so by the choice of $E_{0}$, it is true in $\mathfrak{M}^{*} \uparrow \alpha_{i+1}^{\delta}$; say as exemplified by $\delta^{\prime}$. Let us note that in $L$, we have $\delta<_{L} \delta^{\prime}$ or $\delta^{\prime}<_{L} \delta$, and let us assume that $\delta^{\prime}<_{L} \delta$, as the other case is symmetric. We claim that $\chi_{\left[a_{\delta}^{\prime}\right]}$ exemplifies that $i \in \operatorname{inv}_{\overline{\mathfrak{A}}, \delta}\left(\chi_{\left[a_{\delta}\right]}\right)$. If not, we can find $\bar{g} \in C^{N\left(\mathfrak{A}_{\alpha_{i}^{\delta}}\right)} \backslash C^{0}$ with $0 \leq \bar{g}$ and $g \stackrel{\text { def }}{=} \lim _{n} g_{n} \leq\left|\chi_{\left[a_{\delta}\right]}-\chi_{\left[a_{\delta}^{\prime}\right]}\right|=$ $\chi_{\left[a_{\delta} \Delta a_{\delta}^{\prime}\right]} \leq 1$. By the triangle inequality it follows that

$$
g \leq\left|\chi_{\left[a_{\delta}\right]}-f\right|+\left|f-\chi_{\left[a_{\delta}^{\prime}\right]}\right|
$$

We have that for every $x$ both $\left|\chi_{\left[a_{\delta}\right]}-f\right|(x)$ and $\left|\chi_{\left[a_{\delta}\right]}-f^{\prime}\right|(x)$ are equal to $f(x)$ if $x \in\left[a_{\delta}^{c}\right]$ and $1-f(x)$ if $x \in\left[a_{\delta^{\prime}}\right]$. The possible difference is on $\left[a_{\delta} \backslash a_{\delta^{\prime}}\right]$, where the former function is equal to $1-f(x)$ and the latter to $f(x)$. We now claim that $f$ is constant on $\left[a_{\delta} \backslash a_{\delta^{\prime}}\right]$.

Clearly, it suffices to show that each $f_{n}$ is constant on $\left[a_{\delta}\right]$ $\left.a_{\delta^{\prime}}\right]$, and by the choice of $\left\{\beta_{n}: n<\omega\right\}$, it suffices to show that, for each $l<2$ and each $n, \chi_{\left[a_{\beta_{n}}^{l}\right]}$ is constant on $\left[a_{\delta} \backslash a_{\delta^{\prime}}\right]$. Let $\beta=$ $\beta_{n}$ for some $n$. If $\beta \leq_{L} \delta^{\prime}$, then $a_{\beta} \leq a_{\delta^{\prime}}$ so $\chi_{\left[a_{\beta}\right]}$ is constantly 0 on $\left[a_{\delta} \backslash a_{\delta^{\prime}}\right]$. In addition, we have $a_{\beta}^{c} \geq a_{\delta^{\prime}}^{c} \geq\left(a_{\delta} \backslash a_{\delta}^{\prime}\right)$, so $\chi_{\left[a_{\beta}^{c}\right]}$ is constantly 1 on $\left[a_{\delta} \backslash a_{\delta^{\prime}}\right]$. If $\beta \geq_{L} \delta^{\prime}$, then $\beta \geq_{L} \delta$ by the choice of $\delta^{\prime}$, so $a_{\beta} \geq a_{\delta}$, and hence $\chi_{\left[a_{\beta}\right]}$ is constantly 1 on $\left[a_{\delta} \backslash a_{\delta^{\prime}}\right]$. In addition, $a_{\beta}^{c} \leq a_{\delta}^{c}$ so $\chi_{\left[a_{\beta}^{c}\right]}$ is constantly 0 on $\left[a_{\delta} \backslash a_{\delta^{\prime}}\right]$, and the statement is proved.
Let $\varepsilon \in[0,1]$ be such that $f$ is constantly $\varepsilon$ on $\left[a_{\delta} \backslash a_{\delta^{\prime}}\right]$. Say $\varepsilon \leq 1 / 2$ as the other case is symmetric. Hence $\max \{f, 1-f\}$ on $a_{\delta} \backslash a_{\delta^{\prime}}$ is $1-f$, in particular we have

$$
0 \leq g \leq\left|\chi_{\left[a_{\delta}\right]}-f\right|+\left|f-\chi_{\left[a_{\delta}^{\prime}\right]}\right| \leq 2 \cdot\left|\chi_{\left[a_{\delta}\right]}-f\right|
$$

and hence $1 / 2 \cdot g$ is a function which contradicts that $\bar{f}$ exemplifies that $i \in \operatorname{inv}_{\overline{\mathfrak{A}}, \delta}\left(\chi_{\left[a_{\delta}\right]}\right)$. A contradiction and hence $\chi_{\left[a_{\delta}^{\prime}\right]}$ exemplifies that $i \in \operatorname{inv}_{\overline{\mathfrak{A}}, \delta}\left(\chi_{\left[a_{\delta}\right]}\right)$ as required.

Now suppose that $i \in \operatorname{inv}_{\overline{\mathfrak{A}}, \delta}\left(\chi_{\left[a_{\delta}\right]}\right)$ and assume without loss of generality by Claim 1 that $\bar{f}=\chi_{\left[a_{\delta}^{\prime}\right]}$ for some $\delta^{\prime}$. We need to prove that $i \in \operatorname{inv}_{\overline{\mathfrak{A}}, \delta}\left(a_{\delta}\right)$ as exemplified by $a_{\delta^{\prime}}$. But indeed, if for some $\beta<\alpha_{i}^{\delta}$ and $l<2$, we have that $a_{\beta}^{l} \cap a_{\delta} \neq a_{\beta} \cap$ $a_{\delta^{\prime}} \bmod \mathfrak{\mathfrak { A }}^{\alpha_{i}^{\delta}}$, then there is $w>0$ in $\mathfrak{\mathfrak { A }}^{\alpha_{i}^{\delta}}$ with $w \leq a_{\delta} \Delta a_{\delta^{\prime}}$ and then clearly the sequence $\left\langle\chi_{[w]}, \chi_{[w]}, \ldots\right\rangle$ is not in $C^{0}$ and is below $\left\langle\chi_{\left[a_{\delta} \Delta a_{\delta^{\prime}}\right]}, \chi_{\left[a_{\delta} \Delta a_{\delta^{\prime}}\right]}, \ldots\right\rangle$, a contradiction.

With this, putting together all the sublemmas and the Kojman-Shelah result, we finish the proof of the Construction Lemma.

\section{Conflict of Interests}

The author declares that there is no conflict of interests regarding the publication of this paper.

\section{Acknowledgments}

The author thanks EPSRC for the Grants EP/G068720 and EP/I00498 which supported this research and the University of Wroclaw in Poland for their invitation in October 2010, when some of the preliminary results were presented. The author especially thanks Grzegorz Plebanek for the many productive conversations during the development of this paper.

\section{References}

[1] M. Malliaris and S. Shelah, "General topology meets model theory, on $p$ and $t$," Proceedings of the National Academy of Sciences of the United States of America, vol. 110, no. 33, pp. 13300 13305, 2013.

[2] S. Shelah and A. Usvyatsov, "Banach spaces and groups-order properties and universal models," Israel Journal of Mathematics, vol. 152, pp. 245-270, 2006.

[3] C. Brech and P. Koszmider, "On universal Banach spaces of density continuum," Israel Journal of Mathematics, vol. 190, pp. 93110, 2012.

[4] C. Brech and P. Koszmider, "On universal spaces for the class of Banach spaces whose dual balls are uniform Eberlein compacts," Proceedings of the American Mathematical Society, vol. 141, no. 4, pp. 1267-1280, 2013.

[5] S. Shelah, "No universal group in a cardinal," http://xxx.tau .ac.il/abs/1311.4997v1 .

[6] M. Kojman and S. Shelah, "Nonexistence of universal orders in many cardinals," The Journal of Symbolic Logic, vol. 57, no. 3, pp. 875-891, 1992. 
[7] I. Kaplansky, "Lattices of continuous functions," Bulletin of the American Mathematical Society, vol. 53, pp. 617-623, 1947.

[8] S. Shelah and A. Usvyatsov, "Unstable classes of metricstructures," http://arxiv.org/abs/0810.0734 .

[9] M. Džamonja, "Banach spaces from one Cohen real".

[10] S. Banach, Theorie Des Operations Lineaires, Volume 1 of Monografie Matem-Atyczne, IMPAN, Warszawa, Poland, 1932.

[11] C. C. Chang and H. J. Keisler, Model Theory, vol. 73 of Studies in Logic and the Foundations of Mathematics, North-Holland Publishing, Amsterdam, The Netherlands, 3rd edition, 1990.

[12] I. Gelfand and A. Kolmogorov, "On rings of continuous functions on topo- logical spaces," Soviet Mathematics Doklady, vol. 22, pp. 11-15, 1939 (Russian).

[13] S. Shelah, Classification Theory and the Number of Nonisomorphic Models, vol. 92 of Studies in Logic and the Foundations of Mathematics, North-Holland Publishing, Amsterdam, The Netherlands, 2nd edition, 1990.

[14] S. Shelah, "Advances in cardinal arithmetic," in Finite and Infinite Combinatorics in Sets and Logic, N. W. Sauer, R. E. Woodrow, and B. Sands, Eds., vol. 411 of NATO Advanced Science Institutes Series C: Mathematical and Physical Sciences, pp. 355-383, Kluwer Academic, Dordrecht, The Netherlands, 1993.

[15] G. Plebanek, "On positive embeddings of $C(K)$ spaces," Studia Mathematica, vol. 216, no. 2, pp. 179-192, 2013. 


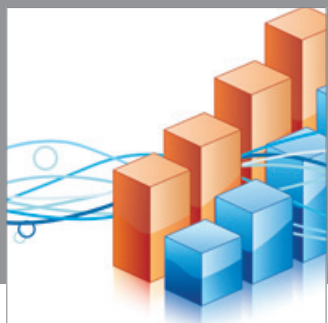

Advances in

Operations Research

mansans

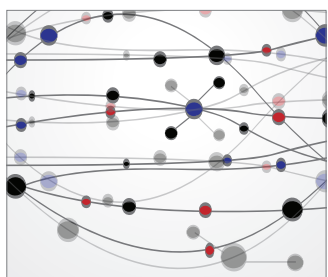

The Scientific World Journal
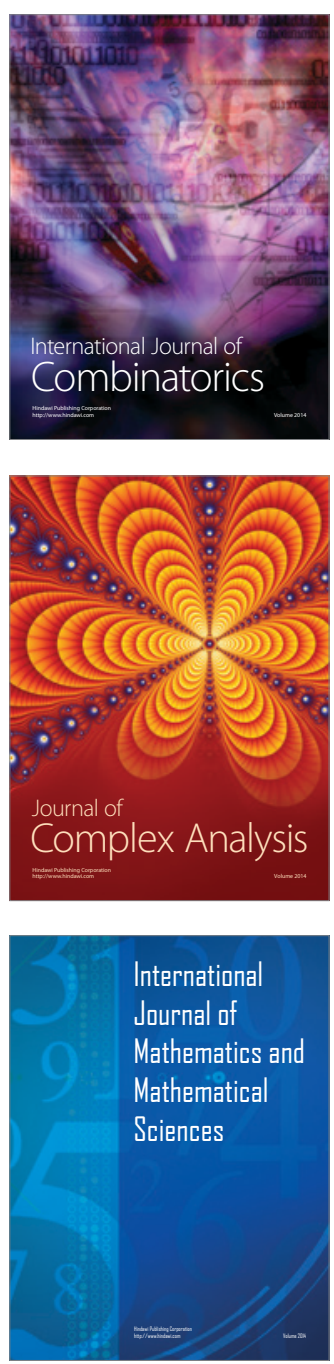
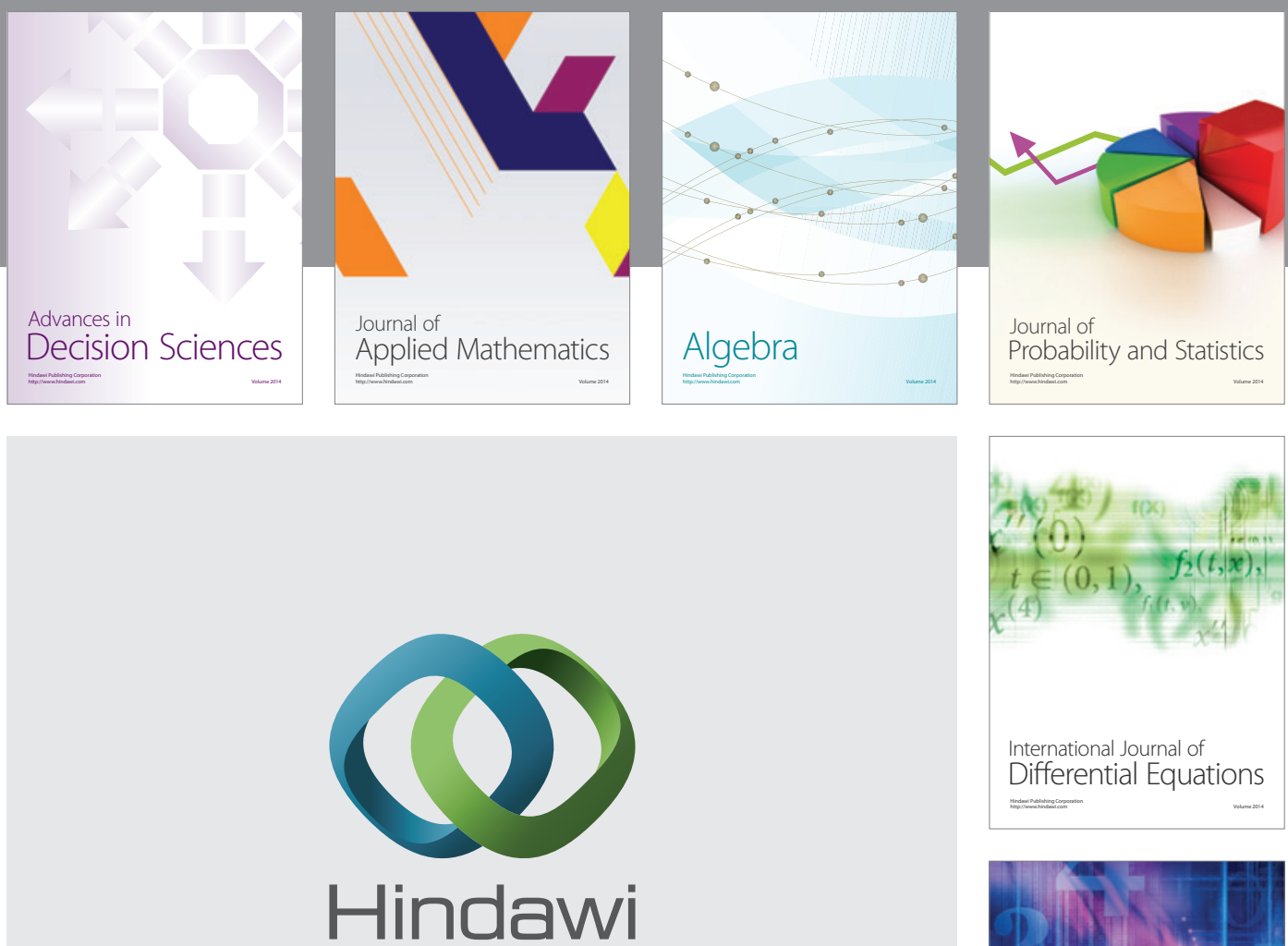

Submit your manuscripts at http://www.hindawi.com
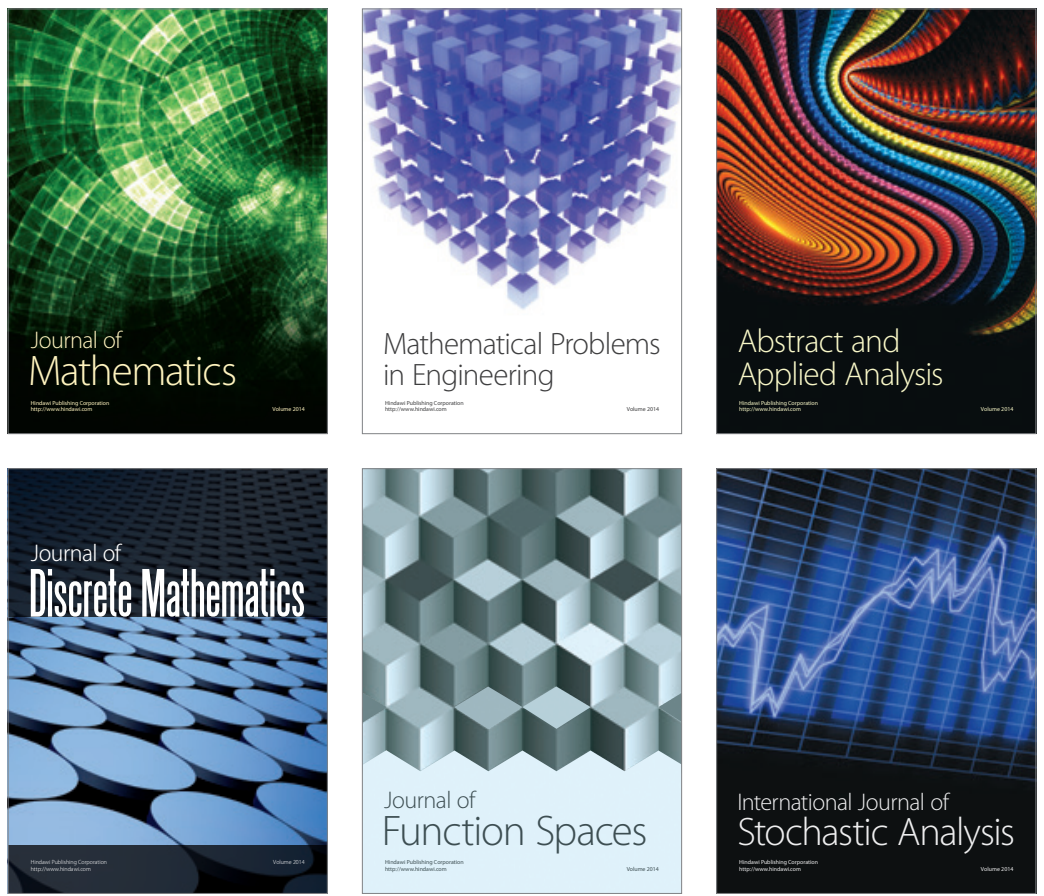

Journal of

Function Spaces

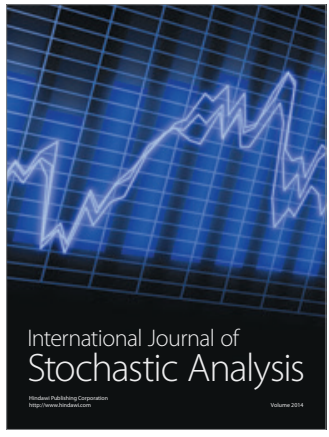

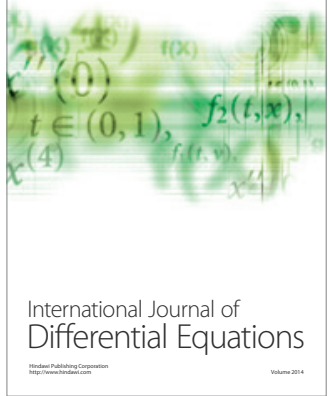
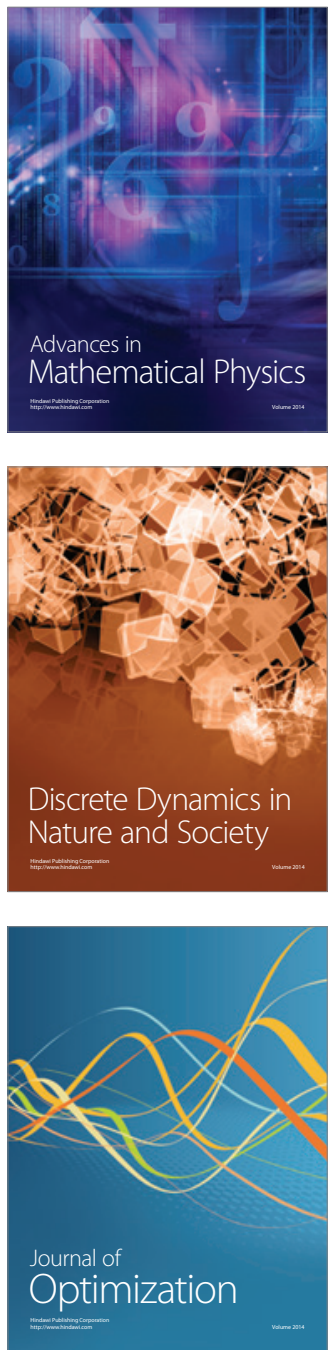